\title{
UNIVERSITÉ LAVAL
}

\author{
MÉMOIRE PRÉSENTÉ À \\ L'UNIVERSITÉ LAVAL \\ COMME EXIGENCE PARTIELLE \\ DE LA MAITRISE EN MÉDECINE EXPÉRIMENTALE \\ VOLET GÉNÉTIQUE \\ OFFERTE À L'UNIVERSITÉ DU QUÉBEC À CHICOUTIMI \\ EN VERTU D'UN PROTOCOLE D'ENTENTE
}

PAR

JOELLE BRASSARD

ÉTUDE CLINIQUE, GÉNÉTIQUE ET ÉPIDÉMIOLOGIQUE DU SYNDROME DE ZELLWEGER ET DE LA CYSTINOSE AU SAGUENAY-LAC-ST-JEAN

MAI 1993

(c) Droits réservés 


\section{Bibliothèque}

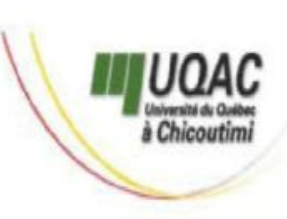

\section{Mise en garde/Advice}

Afin de rendre accessible au plus Motivated by a desire to make the grand nombre le résultat des results of its graduate students' travaux de recherche menés par ses research accessible to all, and in étudiants gradués et dans l'esprit des accordance with the rules règles qui régissent le dépôt et la governing the acceptation and diffusion des mémoires et thèses diffusion of dissertations and produits dans cette Institution, theses in this Institution, the I'Université du Québec à Université du Québec à Chicoutimi (UQAC) est fière de Chicoutimi (UQAC) is proud to rendre accessible une version make a complete version of this complète et gratuite de cette œuvre. work available at no cost to the reader.

L'auteur conserve néanmoins la The author retains ownership of the propriété du droit d'auteur qui copyright of this dissertation or protège ce mémoire ou cette thèse. thesis. Neither the dissertation or Ni le mémoire ou la thèse ni des thesis, nor substantial extracts from extraits substantiels de ceux-ci ne it, may be printed or otherwise peuvent être imprimés ou autrement reproduced without the author's reproduits sans son autorisation. permission. 
Ce mémoire a été réalisé à l'Université du Québec à Chicoutimi dans le cadre du programme de maîtrise en médecine expérimentale (volet génétique) extensionné de l'Université Laval à l'Université du Québec à Chicoutimi. 


\section{RÉSUMÉ}

Le syndrome de Zellweger (syndrome cérébro-hépato-rénal) est une maladie autosomale récessive rare. Dans la région du Saguenay-Lac-St-Jean cette maladie a une incidence particulièrement élevée; en effet depuis 1971 huit cas furent répertoriés, ce qui est de quatre à cinq fois supérieur à l'incidence mondiale. Le phénotype rencontré dans cette région est très semblable à celui décrit dans la littérature. La distribution spatiale des lieux d'origine des parents et des enfants a montré qu'ils étaient presque tous originaires de la région. On a calculé le coefficient moyen de consanguinité qui s'est avéré nul pour les patients et les témoins. Le coefficient de parenté par contre est plus élevé pour les cas que pour les témoins ce qui suggère que le gène est fréquent dans cette population.

La cystinose est une autre maladie autosomale récessive rare qui a au Saguenay-Lac-St-Jean une incidence dix fois supérieure à l'incidence mondiale. Les signes cliniques associés à la cystinose au Saguenay-Lac-St-Jean sont semblables à ceux décrits dans d'autres populations, à l'exception éventuellement d'une atteinte ophtalmique moins sévère. L'étude de la consanguinité et de la parenté ont permis de montrer que le gène est fréquent dans cette population. L'étude de la parenté ainsi que les recherches généalogiques effectuées suggèrent que la présence de cette maladie au Saguenay-Lac-St-Jean est due à un effet fondateur.

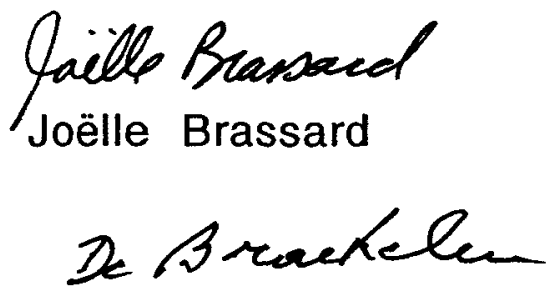

Marc De Braekeleer 


\section{AVANT-PROPOS}

J'ai commencé mes études de deuxième cycle en sachant que cela représenterait un défi des plus intéressant et des plus grand pour moi. J'ai dû pour réussir la rédaction et la présentation de ce mémoire m'appuyer sur un professionnel des plus compétent soit mon directeur de recherche le Dr Marc De Braekeleer. II a cru en moi dès le début et m'appuya depuis ma demande d'inscription jusqu'à la fin de cette aventure. II a su me donner l'amour de la recherche et le désir d'aller plus loin.

Je tiens à remercier le $\mathrm{Dr}$ Jean Larochelle ainsi que tous les pédiatres de la Clinique de pédiatrie de Chicoutimi qui nous ont permis d'avoir accès à leurs dossiers; sans eux cette recherche aurait été impossible à réaliser.

Je remercie tout spécialement mon mari Richard Lévesque pour son aide technique et son précieux support lors de cette recherche. Enfin je remercie mes parents pour la grande disponibilité qu'ils ont fait preuve envers moi et mes enfants et particulièrement ma mère Jacqueline Chiodo qui a su me rendre la vie plus agréable. 
RÉSUMÉ

AVANT-PROPOS

TABLE DES MATIERES.

LISTE DES TABLEAUX

10

LISTE DES FIGURES.

12

INTRODUCTION GÉNÉRALE. 13

\section{PREMIERE PARTIE :}

ÉTUDE CLINIQUE, GÉNÉTIQUE ET ÉPIDÉMIOLOGIQUE DU SYNDROME DE ZELLWEGER AU SAGUENAY-LAC-ST-JEAN.

CHAPITRE I: REVUE DE LA LITTÉRATURE.

I.1 Aspects historiques et cliniques

du syndrome de Zellweger.............................. 19

1.2 Diagnostic prénatal......................................... 20

1.3 Traitement...................................................... 20

1.4 Défaut biochimique......................................... 21

1.4.1 Distributions et propriétés des peroxisomes 
1.4.2 Fonction des peroxisomes.............. 22

1.5 Anatomie pathologique du syndrome

de Zellweger........................................................... 23

1.5.1 Cerveau.................................................. 23

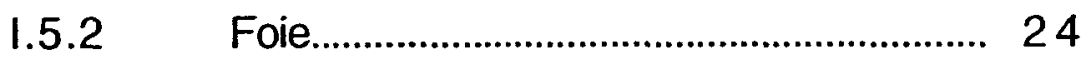

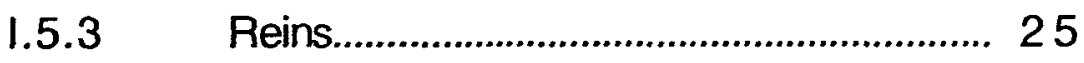

1.5.4 Yeux ........................................................... 25

1.6 Maladies reliées aux désordres

des peroxisomes................................................. 25

$1.7 \quad$ Aspect génétique du syndrome

de Zellweger........................................................... 27

$1.8 \quad$ Épidémiologie.................................................. 31

CHAPITRE II: MATÉRIEL ET MÉTHODES..................................... 33

II.1 Matériel............................................................. 34

II.1.1 Population à l'étude............................. 34

II.2 Méthodologie...................................................... 34

II.2.1 Étude clinique...................................... 34

II.2.2 Étude génétique et

épidémiologique................................... 35

CHAPITRE III: RÉSULTATS...................................................... 37

III.1 Histoires cliniques des enfants atteints.... 38

III.2 Épidémiologie et génétique............................ 44

III.2.1 Incidence du syndrome

de Zellweger au SLSJ.......................... 44 
III.2.2 Distribution spatiale des lieux de naissance des enfants atteints et de leurs parents............ 44

III.2.3 Consanguinité et parenté.................. 47

CHAPITRE IV: DISCUSSION.................................................... 49

CONCLUSION DE LA PREMIERE PARTIE..................................... 54

BIBLIOGRAPHIE DE LA PREMIERE PARTIE................................. 55

DEUXIEME PARTIE:

ÉTUDE CLINIQUE, GÉNÉTIQUE ET ÉPIDÉMIOLOGIQUE DE LA CYSTINOSE AU SAGUENAY-LAC-ST-JEAN.

CHAPTTRE I: REVUE DE LA LITTÉRATURE...............................6 61

I.1 Aspects cliniques et historiques de la cystinose........................................................... 62

I.1.1 Historique............................................62 62

1.1.2 Principales caractéristiques

cliniques.................................................62 62

I.1.3 Manifestations oculaires de la cystinose................................................6 64

I.1.4 Croissance...........................................6 66

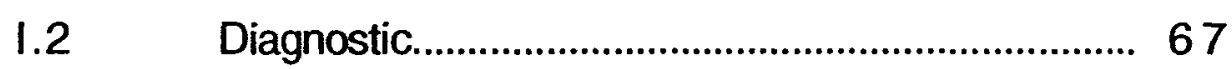




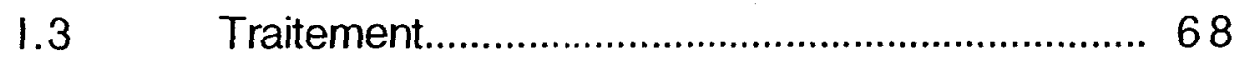

1.4 Défaut biochimique......................................... 70

1.5 Épidémiologie et génétique........................... 72

CHAPITRE II: MATÉRIEL ET MÉTHODES...................................... 75

II.1 Matériel.............................................................. 76

II.1.1 Population à l'étude............................. 76

II.2 Méthodologie............................................................ 76

II.2.1 Étude clinique....................................... 76

II.2.2 Étude génétique

et épidémiologique.............................. 77

CHAPITRE III: RÉSULTATS...................................................... 79

III.1 Histoires cliniques des enfants atteints... 80

III.2 Épidémiologie et génétique............................ 94

III.2.1 Incidence de la cystinose au

SLSJ.......................................................... 94

III.2.2 Distribution spatiale des lieux de naissance des enfants atteints et de leurs parents............ 94

III.2.3 Consanguinité et parenté................... 99

CHAPITRE IV: DISCUSSION.......................................................... 101

CONCLUSION DE LA DEUXIEME PARTIE........................................ 108 
BIBLIOGRAPHIE DE LA DEUXIEME PARTIE................................ 110

CONCLUSION GÉNÉRALE............................................................. 114 


\section{LISTE DES TABLEAUX}

PREMIERE PARTIE

CHAPITRE I

Tableau 1.1 Classification des maladies reliées

aux désordres des peroxisomes

selon l'altération des fonctions

peroxisomales..................................................... 28

Tableau 1.2 Maladies ( autres que le Zellweger)

reliées aux désordres des peroxisomes

et leurs principales caractéristiques......... 29

CHAPITRE III

Tableau 1.3 Caractéristiques cliniques plus

fréquemment rencontrées chez les

enfants atteints du syndrome de

Zellweger au Saguenay-Lac-St-Jean............

Tableau 1.3.A Caractéristiques cliniques moins

fréquemment rencontrées chez les

enfants atteints du syndrome de

Zellweger au Saguenay-Lac-St-Jean...........

Tableau 1.4 Coefficients moyens de consanguinité

et de parenté chez les enfants atteints

du syndrome de Zellweger et des groupes-

témoins au Saguenay-Lac-St-Jean............... 48 
CHAPITRE IV

Tableau 1.5 Comparaison entre les caractéristiques trouvées dans la littérature et celles des patients du Saguenay-Lac-St-Jean............. 52

\section{DEUXIEME PARTIE}

CHAPITRE III

Tableau 2.1 Caractéristiques cliniques rencontrées chez 8 des 9 enfants atteints de cystinose au Saguenay-Lac-St-Jean............ 92

Tableau 2.2 Distribution des lieux d'origine des fondateurs communs aux porteurs obligatoires.

Tableau 2.3 Distribution des lieux d'origine des ancêtres communs aux porteurs obligatoires. 98

Tableau 2.4 Coefficients moyens de consanguinité et de parenté chez les enfants atteints de cystinose et des groupes-témoins au Saguenay-Lac-St-Jean............................. 100

CHAPITRE IV

Tableau 2.5 Comparaison entre les caractéristiques cliniques trouvées dans la littérature et celles des patients du SaguenayLac-St-Jean 


\section{LISTE DES FIGURES}

\section{PREMIERE PARTIE}

CHAPITRE I

Figure 1.1 Localisation de la région du Saguenay-

Lac-St-Jean au Québec.......................................

CHAPITRE III

Figure 1.2 Pédigrees des enfants atteints du

syndrome de Zellweger au Saguenay-

Lac-St-Jean........................................................... 39

Figure 1.3 Distribution des lieux de naissance des

enfants et des porteurs obligatoires du syndrome de Zellweger au Saguenay-

Lac-St-Jean......................................................... 45

\section{DEUXIEME PARTIE}

CHAPITRE III

Figure 2.1 Distribution des lieux de naissance des

enfants et des porteurs obligatoires

de cystinose du Saguenay-Lac-St-Jean...... 95 


\section{INTRODUCTION GÉNÉRALE}

Le Saguenay-Lac-St-Jean (SLSJ) est une région géographiquement isolée qui connaît une haute fréquence de maladies héréditaires à transmission autosomale récessive (Figure 1.1). La diffusion de certains gènes délétères a été favorisée par plusieurs facteurs démographiques, historiques et sociaux qui commencent à peine à être mis en lumière (Bouchard et De Braekeleer 1991).

Depuis les premières recherches du docteur Jean Larochelle sur la tyrosinémie en 1966, les médecins de la région sont plus sensibilisés au problème des maladies héréditaires au SLSJ. C'est par leur intermédiaire que l'on peut repérer des concentrations de maladies rares qui autrement passeraient inaperçues.

Le syndrome de Zellweger qui fait l'objet de la première partie de cette recherche, est l'une de ces maladies. Depuis 1978, six cas ont été investigués à la Clinique de Pédiatrie et à l'hôpital de Chicoutimi. Cette maladie rare a été peu étudiée, la documentation qui traite de ce sujet est par le fait même limitée. L'intérêt pour cette maladie ne s'en trouve pas pour autant réduit, au contraire cela nous donne l'occasion d'explorer les aspects cliniques, génétiques et épidémiologiques des phénotypes rencontrés au SLSJ. La comparaison avec les autres cas rapportés dans la littérature nous permettra peut-être de mettre en lumière les différents aspects de ce syndrome et de tracer un portrait plus juste des cas spécifiques à la région.

Cependant, lors de la réalisation de cette étude, il est devenu évident qu'un petit nombre seulement de familles avaient été 
identifiées. Dès lors, il a été convenu avec mon directeur de recherche de compléter mon mémoire par l'étude d'une autre maladie autosomale récessive, elle aussi peu connue au SLSJ, à savoir la cystinose.

Dans la deuxième partie de cette recherche il sera donc question de la cystinose qui connaît une incidence élevée dans la région. Les aspects cliniques, génétiques et épidémiologiques de cette maladie seront également étudiés. Nous tenterons à l'aide des données recueillies de confirmer la présence d'un effet fondateur. 
FIGURE 1.1 :Localisation de la région du Saguenay-Lac-St-Jean au Québec. 


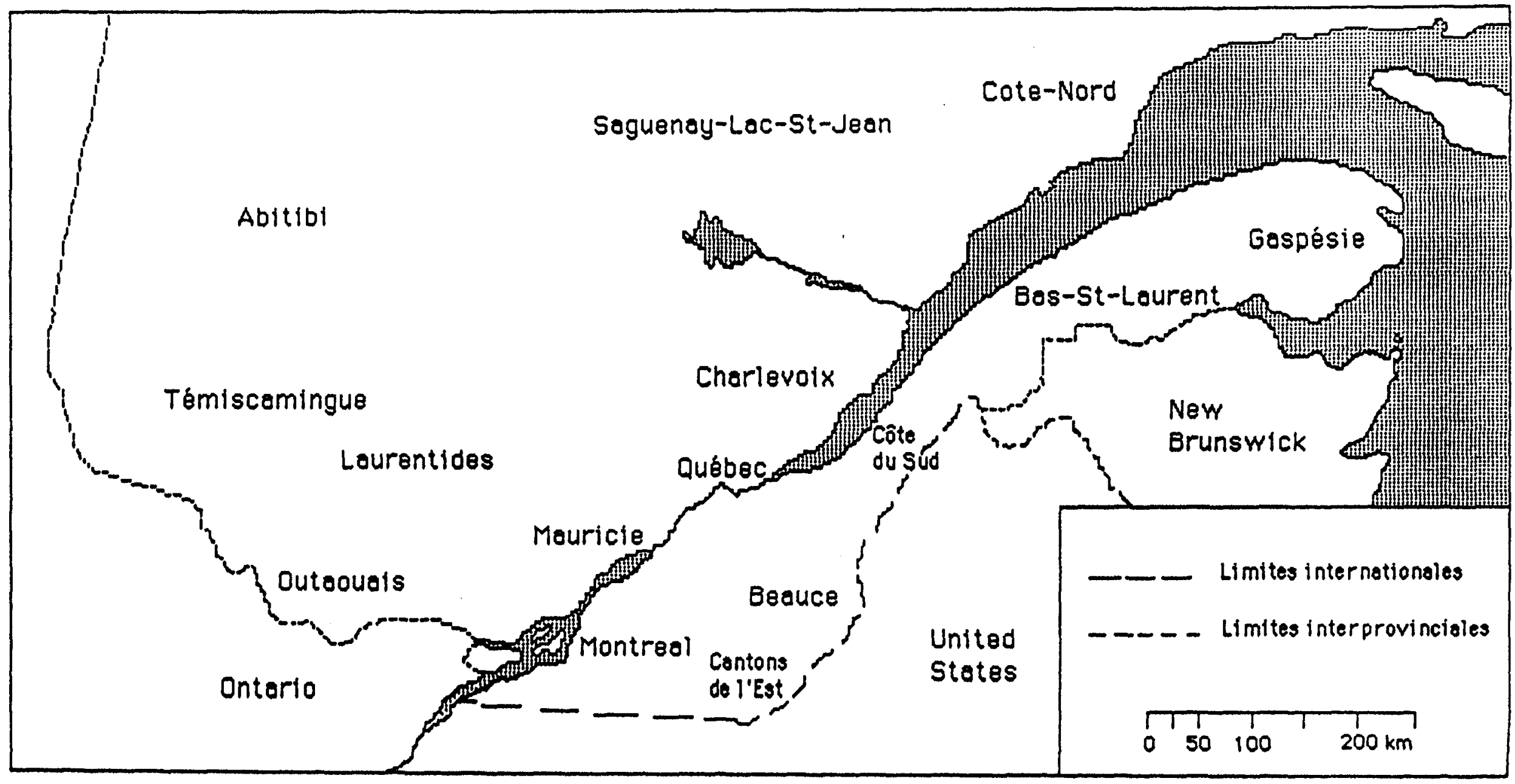




\section{PREMIERE PARTIE}

ÉTUDE CLINIQUE, GÉNÉTIQUE ET ÉPIDÉMIOLOGIQUE DU SYNDROME DE ZELLWEGER AU SAGUENAY-LAC-STJEAN. 
CHAPITRE I

REVUE DE LA LITTÉRATURE 


\section{I.1 Aspects historiques et cliniques du Syndrome de Zellweger}

Le syndrome cérébro-hépato-rénal de Zellweger est une maladie autosomale récessive. Elle fut désignée sous l'appellation de syndrome de Zellweger par Opitz en 1969, en reconnaissance à Hans Zellweger pour le rôle qu'il a joué dans l'identification de la maladie (Opitz 1985). Tout d'abord classée dans la catégorie des anomalies congénitales multiples, cette maladie fut réassignée par Goldfisher et al. en 1973 dans la catégorie des troubles du métabolisme (après qu'ils eurent démontré que les patients avaient une déficience des peroxisomes dans le foie et les reins).

Les principaux signes cliniques sont une hypotonie sévère, un front haut, des fontanelles larges, des opacités cornéennes, de multiples contractures aux articulations, une hépatomégalie, une dyschondroplasie ponctuée et des reins polykystiques. La mort survient généralement dans les six premiers mois de la vie (Govaerts et al. 1982).

Pour le diagnostic, l'examen clinique est un bon indice puisque les patients présentent à peu près tous un faciès très caractéristique; le diagnostic est confirmé par une biopsie hépatique qui démontre l'absence de peroxisomes. L'absence ou un nombre restreint de peroxisomes peut être observé dans des cultures de fibroblastes de la peau des patients atteints (Roels et al. 1987). 


\subsection{Diagnostic prénatal}

Une variété de techniques sont utilisées pour dépister un désordre des peroxisomes. On peut démontrer la présence d'acides gras à longue chaîne et l'incapacité à les oxyder dans les cultures d'amniocytes ou à partir de biopsies chorioniques (Moser et al. 1984; Rocchiccioli et al. 1987; Wanders et al. 1987). On peut également rechercher une déficience au niveau de la synthèse des plasmalogènes (Schutgens et al. 1985; Hajra et al.1985).

\subsection{Traitement}

Au niveau du traitement le potentiel est très limité, les malformations congénitales étant multiples et complexes. L'administration de clofibrate n'augmente pas le nombre de peroxisomes hépatiques (Lazarow et al. 1985; Bjorkhem et al. 1985).

Des éthers lipides ont été administrés par voie orale chez des patients présentant un tableau clinique moins sévère, mais sans grands résultats (Wilson et al. 1986; Holmes et al.1987). Le taux de plasmalogènes dans les globules rouges peut être partiellement normalisé, et le taux d'acides gras en partie diminué par une diète spéciale (Moser et al. 1989). 


\subsection{Défaut biochimique}

\subsubsection{Distributions et propriétés des peroxisomes}

Les peroxisomes sont présents dans la plupart sinon toutes les cellules des mammifères, à l'exception des érythrocytes matures. Leur grosseur et leur nombre varient considérablement. Mais c'est surtout dans le foie et les reins que les peroxisomes sont en plus grand nombre. Les peroxisomes dans ces tissus apparaissent au microscope électronique, ronds ou ovales, entourés d'une membrane d'environ 6,5 à $7 \mathrm{~nm}$ d'épaisseur. Le diamètre est d'environ $0,5 \mu \mathrm{m}$ (il peut varier entre 0,2 et $1 \mu \mathrm{m}$ ) (Hruban et Rechcigl,1969). Ils ne contiennent pas d'ADN ni de glycoprotéines.

Les peroxisomes sont abondants dans les cellules excrétrices de stéroïdes, comme le cortex surrénalien; ils se rencontrent aussi dans les cellules des glandes sébacées, les cellules de myéline et plusieurs types de cellules de l'appareil digestif. Dans certaines cellules comme les glandes sébacées, les peroxisomes ne se présentent pas comme des sphères individuelles, mais plutôt comme une interconnection dans laquelle il y aurait un compartiment intracellulaire contorsionné et élaboré, donnant l'impression d'un “réticulum peroxisomal" (Lazarow et al.1980). 


\subsubsection{Fonctions des peroxisomes}

Des études biochimiques ont montré que les peroxisomes sont impliqués dans plusieurs fonctions cataboliques et anaboliques, comme par exemple la synthèse des plasmalogènes, du cholestérol et des acides biliaires; ils sont aussi impliqués dans la glucogénèse.

Au niveau catabolique les peroxisomes sont importants pour la dégradation de l'acide pipécolique, l'acide phytanique, les acides gras à longue chaîne ainsi que le raccourcissement de la chaîne insaturée d'acyl-coA .

Toutes les réactions de B-oxydation peroxisomale des acides gras s'effectuent dans le foie, les reins, l'intestin, les poumons, la rate, les muscles, le tissu adipeux, le coeur et le cortex surrénalien. II est possible d'augmenter la ß-oxydation hépatique des peroxisomes, par une diète riche en acide gras ou par des médicaments hypolipodémiants (Lazarow et Moser, 1989 ).

Les peroxisomes contiennent un groupe d'enzymes qui utilisent l'oxygène pour oxyder une variété de substrats et ainsi former le peroxide d'hydrogène. De Duve a nommé les peroxisomes justement parce qu'ils ont des peroxides qui sont à la base de la respiration (DeDuve et Baudhuin,1966).

La respiration peroxisomale est estimée à $20 \%$ de l'oxygène consommé par le foie. Une dernière fonction de la respiration peroxisomale semble être la contribution à la thermogénèse, ce qui a été démontré dans des études sur les peroxisomes dans les tissus adipeux, lors de l'adaptation au froid (Ahlabo et Barnard,1971). 


\section{I.5 Anatomie pathologique du syndrome de Zellweger}

Goldfisher et al.(1973) ont rapporté que les patients atteints du syndrome de Zellweger présentaient une absence de peroxisomes dans le foie et les reins. Toutes les autres études ont confirmé cette observation. L'absence de peroxisomes dans le foie est un critère essentiel pour le diagnostic du syndrome de Zellweger. Les peroxisomes sont aussi réduits en nombre ou absents dans les cultures de fibroblastes de la peau des personnes atteintes (Lazarow et al. 1988).

\section{I.5.1 Cerveau}

L'anomalie la plus importante et surtout la plus intrigante est le désordre de migration des neurones. Ce désordre entraine des caractéristiques particulières au niveau cytoarchitectural des hémisphères cérébraux du cervelet et des noyaux olivaires inférieurs. Selon Evrad et al. (1978) le mécanisme de migration des neurones serait troublé à partir du troisième mois de gestation; des études effectuées par Powers et al. en 1985 avec des foetus atteints du syndrome de Zellweger l'ont confirmé.

Dans l'hémisphère cérébral, les neurones qui sont normalement destinés à aller à la périphérie de la couche corticale sont distribués à l'intérieur de cette couche corticale et dans la matière blanche sous-jacente. Cette déficience de migration amène des circonvolutions cérébrales qui peuvent être anormalement 
petites (microgyrie) ou épaisses (pachygyrie) (Lazarow et Moser, 1989).

Les autres traits caractéristiques sont le défaut de migration affectant les cellules de Purkinje dans le cervelet et une discontinuité laminaire dans les principaux noyaux du complexe olivaire inférieur. Les patients atteints du syndrome de Zellweger présentent aussi une hypoplasie du bulbe olfactif et une dystrophie neuroaxonale du noyau dorsal de Clark et du noyau latéral cunéate (Powers, 1987; Deleon,1977).

En plus de ces défauts, Passarge et McAdams en 1967 ont noté une sévère déficience en myéline et une accumulation de lipides sudanophiliques. Une déficience générale de myéline et un processus actif de démyélinisation, ont aussi été notés (Agamanolis et al. 1976).

\subsubsection{Foie}

Le foie des personnes atteintes du syndrome de Zellweger apparaît très souvent hypertrophié et fibreux. Plusieurs patients présentent une cirrhose micronodulaire et une choléstase est présente chez plus de la moitié des cas étudiés (Heymans, 1984). Ces anomalies sont plus sévères chez les enfants plus âgés. Des dépôts excessifs de fer ont été rapportés, mais ces dépôts diminuent avec le temps et redeviennent dans les limites de la normale chez les enfants de plus de vingt semaines (Vitale et al.1969; Gilchrist et al. 1976). 


\subsubsection{Reins}

Des kystes ont été trouvés chez presque tous les enfants étudiés. Leur taille varie du micro-kyste glomérulaire au large kyste cortical du glomérule et du tubule (Heymans 1984). Les kystes sont présents chez tous les foetus observés (Powers et al. 1985).

\subsubsection{Yeux}

Les anomalies retrouvées au niveau des yeux sont importantes et se situent dans le segment antérieur et postérieur de l'oeil. Ces anomalies comportent: des opacités cornéennes, une cataracte et un glaucome congénital, une perte de cellules ganglionnaires, une gliose du nerf optique, une atrophie optique et des changements des pigments de la rétine et de l'épithélium (Cohen et al. 1984).

\section{I.6 Maladies reliées aux désordres des peroxisomes}

La relation entre le syndrome de Zellweger et les autres maladies reliées aux désordres des peroxisomes est difficile à cerner. La différence majeure au niveau clinique résiderait dans une gradation de la sévérité de la maladie. Le syndrome de Zellweger serait la plus sévère, la maladie de Refsum, la moins sévère et l'adrénoleucodystrophie serait intermédiaire. Pour des raisons variées, il semblerait que l'acidémie hyperpipécolique soit plus 
difficile à séparer phénotypiquement du syndrome de Zellweger ou de l'adrénoleucodystrophie néonatale ( Aldebert et al. 1989)

Aldebert et al (1989) ont identifié six génotypes distincts relevant d'un désordre des peroxisomes; ils ont comparé les génotypes avec les phénotypes. Les conclusions ne se sont pas avérées significatives, car les corrélations sont représentées dans des groupes de complémentation différents; ainsi le phénotype le plus commun est dispersé dans cinq des six groupes. Ces conclusions indiquent que la base de la nomenclature ne réussit pas à décrire ou définir le génotype.

Par ailleurs selon d'autres chercheurs, ce groupe de maladie peut être divisé en trois catégories (Tager et al. 1990) (Tableau 1.1). Ce sont:

1- Maladies dans lesquelles les peroxisomes sont virtuellement absents. Dans cette catégorie on peut classer le syndrome de Zellweger, la maladie infantile de Refsum, l'acidémie hyperpicolique et l'adrénoleucodystrophie néonatale.

2- Maladies dans lesquelles les peroxisomes sont présents mais plusieurs fonctions peroxisomales sont altérées. Dans cette catégorie on retrouve la forme rhizomélique de dyschondroplasie ponctuée et le "Zellweger-like syndrome".

3- Maladies dans lesquelles une seule fonction peroxisomale est altérée. Dans cette catégorie on retrouve l'adrénoleucodystrophie liée à $X$, la maladie de Refsum, l'acatalasémie, la déficience en thiolase peroxisomale, la déficience en Acyl-CoA oxidase et l'hyperoxalurie de type I (Tager et al. 1990). 
Les principales caractéristiques de ces maladies sont présentées dans le tableau 1.2.

\subsection{Aspect génétique du syndrome de Zellweger}

Le peroxisome est une structure complexe et plusieurs gènes sont impliqués pour son fonctionnement; il y en aurait cinq ou même six d'impliqués (Tager et al. 1990). Par contre il reste à savoir si les différents phénotypes observés sont le résultat de mutations différentes d'un même gène ou bien le résultat de différentes mutations dans chacun des gènes codant pour l'assemblage du peroxisome. Les deux hypothèses sont plausibles car on sait que des mutations différentes d'un même gène peuvent amener des maladies cliniquement distinctes et que des mutations dans des gènes différents peuvent amener des phénotypes cliniques à peu près semblables.

Le syndrome de Zellweger, qui est considéré comme le prototype des maladies du groupe des désordres des peroxisomes, est reconnu comme ayant un mode de transmission autosomal récessif, mais aucun gène spécifique n'a encore été identifié. Par contre un groupe de chercheurs japonais ont rapporté le cas d'une patiente atteinte du syndrome de Zellweger et présentant une micro-délétion du chromosome 7 (Naritomi et al. 1988). 
TABLEAU 1.1

Classification des maladies reliées aux désordres des peroxisomes selon l'altération des fonctions peroxisomales

\begin{tabular}{|c|c|c|}
\hline $\begin{array}{l}\text { Catégorie } 1 \\
\text { Peroxisomes absents }\end{array}$ & $\begin{array}{l}\text { Catégorie } 2 \\
\text { Plusieurs fonctions } \\
\text { peroxisomales } \\
\text { altérées }\end{array}$ & $\begin{array}{l}\text { Catégorie } 3 \\
\text { Une seule fonction } \\
\text { peroxisomale altérée }\end{array}$ \\
\hline $\begin{array}{l}\text { Syndrome de } \\
\text { Zellweger }\end{array}$ & $\begin{array}{l}\text { Forme rhizomélique } \\
\text { de dyschondroplasie } \\
\text { ponctuée }\end{array}$ & $\begin{array}{l}\text { Adrénoleucodystro- } \\
\text { phie liée au } \\
\text { chromosome } x\end{array}$ \\
\hline $\begin{array}{l}\text { Maladie infantile de } \\
\text { Refsum }\end{array}$ & $\begin{array}{l}\text { "Zellweger-like } \\
\text { syndrome" }\end{array}$ & Maladie de Refsum \\
\hline $\begin{array}{l}\text { Acidémie } \\
\text { hyperpipécolique }\end{array}$ & & Acatalasémie \\
\hline \multirow[t]{3}{*}{$\begin{array}{l}\text { Adrénoleucodystro- } \\
\text { phie néonatale }\end{array}$} & & $\begin{array}{l}\text { Déficience en } \\
\text { thiolase } \\
\text { peroxisomale }\end{array}$ \\
\hline & & $\begin{array}{l}\text { Déficience en Acyl- } \\
\text { CoA oxidase }\end{array}$ \\
\hline & & $\begin{array}{l}\text { Hyperoxalurie de } \\
\text { type } 1\end{array}$ \\
\hline
\end{tabular}

D’après Tager et al. (1990). 
TABLEAU 1.2

Maladies (autres que le Zellweger) reliées aux désordres des peroxisomes et leurs principales caractéristiques

\begin{tabular}{|l|l|}
\hline Nom de la maladie & $\begin{array}{l}\text { Principales caractéristiques } \\
\text { adrénoleucodystrophie liée }\end{array}$ \\
\hline $\begin{array}{l}\text { Désordre autosomal récessif lié au } \\
\text { chromosone X. Détérioration } \\
\text { progressive de la vision, des } \\
\text { fonctions intellectuelles et motrices, } \\
\text { hypofonctionnement du cortex } \\
\text { surrénalien. La mort survient } \\
\text { généralement durant l'adolescence. }\end{array}$ \\
\hline Maladie de Refsum & $\begin{array}{l}\text { Désordre autosomal récessif. } \\
\text { Accumulation d'acide gras méthylé et } \\
\text { d'acide phytanique. Dégénération de la } \\
\text { rétine, neuropathie périphérique et } \\
\text { altération de l'ouie. Affecte les } \\
\text { adultes. }\end{array}$ \\
\hline \multirow{2}{*}{$\begin{array}{l}\text { Acatalasémie } \\
\text { Megrés variés de déficience de la } \\
\text { catalase. Pas de lésions } \\
\text { pathologiques sauf pour des lésions } \\
\text { de gangrène orale. }\end{array}$} \\
\hline $\begin{array}{l}\text { Désordre autosomal récessif. } \\
\text { Seulement 10 patients rapportés, } \\
\text { âgés entre 33 mois et 10 ans et tous } \\
\text { de sexe mâle. Retard mental, } \\
\text { hypotonie, dysfonction neurologique } \\
\text { progressive, fibrose hépatique, } \\
\text { rétinopathie et dysmorphisme mineur. }\end{array}$
\end{tabular}


TABLEAU 1.2 (suite)

\begin{tabular}{|c|c|}
\hline Acidémie hyperpipécolique & $\begin{array}{l}\text { Désordre autosomal récessif. } \\
\text { Seulement } 4 \text { patients rapportés, tous } \\
\text { de sexe mâle. Retard mental, } \\
\text { hypotonie, dysfonction neurologique } \\
\text { progressive, fibrose hépatique, } \\
\text { rétinopathie et dysmorphisme } \\
\text { mineur. Trois des } 4 \text { patients sont } \\
\text { décédés dans la seconde année de vie. }\end{array}$ \\
\hline $\begin{array}{l}\text { Adrénoleucodystrophie } \\
\text { néonatale }\end{array}$ & $\begin{array}{l}\text { Désordre autosomal récessif. Au } \\
\text { moins } 20 \text { patients décrits. Les } \\
\text { premiers symptômes apparaissent } \\
\text { dans la première année de vie. } \\
\text { Dysmorphisme du crane, sévère } \\
\text { hypotonie, convulsions, retard } \\
\text { psychomoteur et staturo-pondéral, } \\
\text { démyélinisation du cerveau, } \\
\text { polymicrogyrie, hypofonctionnement } \\
\text { du cortex surrénalien dans quelques } \\
\text { cas. Généralement la mort survient } \\
\text { avant l'âge de six ans. }\end{array}$ \\
\hline $\begin{array}{l}\text { Dyschondroplasie ponctuée } \\
\text { de type rhizomélique }\end{array}$ & $\begin{array}{l}\text { Désordre récessif. Petitesse } \\
\text { disproportionnée affectant les } \\
\text { parties proximales des extrémités. } \\
\text { Faciès typique, atteinte oculaire, } \\
\text { retard mental. La mort survient dans } \\
\text { l'enfance. }\end{array}$ \\
\hline
\end{tabular}

D'après Schutgens et al. (1986) 
Le cas en question est celui d'une petite Japonaise âgée de seize jours, née après trente-huit semaines de gestation. A la naissance elle souffrait d'une sévère hypotonie et de plusieurs autres symptomes ressemblant à ceux du syndrome de Zellweger. Le diagnostic de Zellweger fut confirmé par une biopsie de la peau et du rectum, ainsi qu'une biopsie hépatique montrant une déficience marquée en peroxisomes (Naritomi et al. 1988). Des investigations cytogénétiques furent entreprises et une micro-délétion du segment proximal du bras long du chromosome 7 fut observée dans toutes les cellules en pro-métaphase. Le karyotype de la patiente fut désigné : 46,XX,del. (7) (q11.21 q11.23). Le karyotype des parents était normal (Naritomi. et al.1988).

Un cas avec une délétion similaire a été rapporté par Fryman et al. en 1986. Il s'agit d'un garçon âgé de quatorze ans présentant des caractéristiques ressemblant au syndrome de Zellweger, mais dont le diagnostic est incertain. Ce qu'il y a de commun entre ces deux cas est le fait qu'ils avaient tous les deux un long philtrum et un retard de croissance in utero, ce qui pourtant est rare dans le syndrome de Zellweger (Naritomi et al. 1988).

\subsection{Epidémiologie}

L'incidence du syndrome de Zellweger est de 1 pour 100,000 naissances. La fréquence des maladies dues à une déficience des peroxisomes est évaluée de 1 pour 25000 à 1 pour 50000 naissances vivantes. En cinq ans, 207 patients avec un désordre des peroxisomes ont été identifiés et étudiés au Kennedy Institute; $69 \%$ 
venaient des Etats-Unis et $22 \%$ du Canada. Pour environ 3,6 millions de naissances par année aux Etats-Unis, le Kennedy Institute à lui seul compte pour une incidence de 0,79 cas par 100000 naissances aux Etats-Unis (Lazarow et Moser,1989). 


\section{CHAPITRE II}

MATÉRIELET MÉTHODES 


\section{II.1. Matériel}

\section{II.1.1 Population à l'étude}

Suite à la naissance d'un enfant atteint du syndrome de Zellweger en 1990, les docteurs Jean Larochelle et Charles Morin de la Clinique de Pédiatrie de Chicoutimi ont contacté le docteur Marc De Braekeleer pour l'informer qu'il y avait déjà eu d'autres cas similaires. Ils lui fournirent la liste des patients atteints et, après autorisation du directeur des services professionnels de l'hôpital de Chicoutimi et de l'ensemble des pédiatres de la Clinique de Pédiatrie, les dossiers ont été consultés à la Clinique de Pédiatrie et aux archives de l'hôpital de Chicoutimi. II faut signaler qu'une enquête des pédiatres de Chicoutimi auprès de leurs confrères de la région du SLSJ n'a pas révélé d'autres cas de syndrome de Zellweger.

\section{II.2. Méthodologie}

\section{II.2.1 Étude clinique}

Les données pertinentes ont été extraites des dossiers, à savoir: la date et le lieu de naissance des patients ainsi que le lieu de résidence de leurs parents, le poids et la taille à la naissance, la description clinique, les différentes hospitalisations, l'évolution de la maladie, la date de décès ainsi que les résultats d'autopsie. 


\subsubsection{Etude génétique et épidémiologique}

La méthodologie utilisée est la même que celle utilisée pour d'autres maladies récessives au SLSJ. Elle a été décrite en détails dans De Braekeleer et Larochelle (1990).

L'incidence à la naissance a été calculée pour la période 1971-1990 en divisant le nombre de naissances d'enfants atteints nés au SLSJ par le nombre de naissances dans la région pendant cette période. Le taux de porteurs a été estimé en utilisant la loi d'Hardy Weinberg (De Braekeleer et Larochelle, 1990).

Les lieux de résidence des parents au moment de la naissance des porteurs obligatoires du syndrome de Zellweger (grands-parents des enfants atteints) ont été extraits du fichier de population du SLSJ développé et conservé à SOREP. Ce fichier de population contient plus de 900000 actes de naissance, mariage et décès relevé de la population française catholique du SLSJ allant de 1838 à 1986 (De Braekeleer et Larochelle,1990).

Les généalogies ont été automatiquement reconstituées à l'aide de MEDIC 4, un programme développé à SOREP. Le coefficient de consanguinité, c'est-à-dire la probabilité que les deux gènes qu'un individu possède à un locus soient identiques par descendance, a été calculé par le programme CONB développé à SOREP; quant au coefficient de parenté, il a été calculé par le programme APPB également développé à SOREP (Jacquard, 1974). 
Trois groupes-témoins ont été créés à l'aide du fichier de population. Ils ont été sélectionnés sur la base du lieu et de la date de mariage (à \pm trois mois près) des parents des enfants atteints, ainsi que sur le statut socio-économique des couples, préalablement définis par la profession de l'homme. Ces trois groupes-témoins ont été utilisés pour l'étude de la consanguinité et de la parenté (De Braekeleer et Larochelle, 1990). 
CHAPITRE III

RÉSULTATS 


\section{III.1 Histoires cliniques des enfants atteints}

Cas No. 1 (Figure 1.2)

Il s'agit d'une fille née par césarienne. A la naissance on nota une hypotonie sévère, une micrognathie, un palais ogival, un épicanthus et une absence des réflexes de Moro et de succion; le réflexe de préhension était faible. Elle a également présenté des convulsions durant l'évolution de la maladie. Le caryotype était normal. Le diagnostic a été confirmé à la biopsie hépatique. Le décès dû à une acidose métabolique et à une déshydratation est survenu à 17 semaines. L'autopsie a été refusée. L'histoire familiale révéla que deux autres enfants de cette fratrie étaient décédés à quelques mois du syndrome de Zellweger ( No.2 et No.3).

Le premier enfant de sexe masculin (No.2) présentait un dysmorphisme généralisé, une hypotonie sévère ainsi que des opacités cornéennes.

Le deuxième enfant de sexe féminin (No.3), présentait une hypotonie, une aréflexie, des convulsions ainsi qu'un dysmorphisme. L'autopsie a montré la présence de multiples kystes rénaux, une hémosidérose marquée du foie et de la rate ainsi qu'un ramollissement cavitaire bilatéral de la zone périventriculaire du cerveau. La famille ayant été perdue de vue, aucune autre information concernant ces deux enfants n'a pu être obtenue. 


\section{Figure 2}

Pédigrees des enfants atteints du syndrome de Zellweger au SLSJ

$\mathrm{H}^{*} 1-\mathrm{N}^{*} 2-\mathrm{N}^{*} 3$

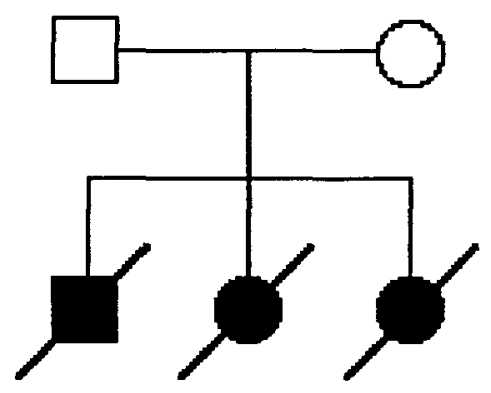

$\mathrm{N}^{*} 5$

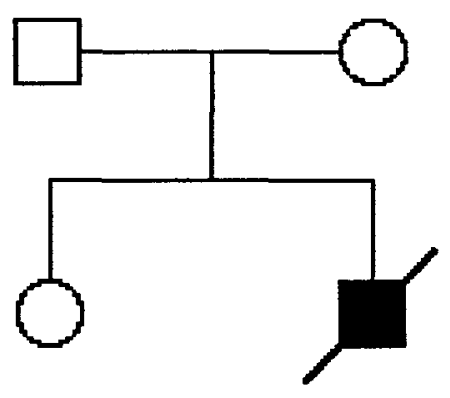

$N^{*} 4$

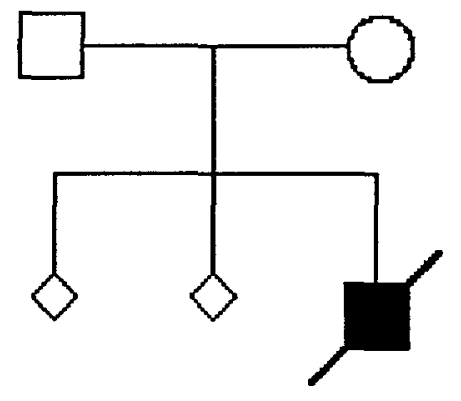

$\mathrm{N}^{*} 6$

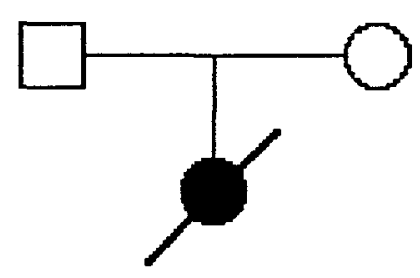


Cas No. 4 (Figure 1.2)

II s'agit d'un garçon né d'un accouchement normal. A la naissance l'enfant était cyanosé; on nota des fontanelles larges, un front fuyant, de l'hypertélorisme, un palais ogival et une micrognathie. L'enfant était hypotonique et présentait de l'aréflexie et une absence du réflexe de Moro. La radiographie des genoux montra une chondrocalcinose au niveau des rotules. Durant l'évolution de la maladie, il a présenté des convulsions. Le décès, dû à une surinfection pulmonaire, est survenu à 5 semaines; l'autopsie a montré des reins microkystiques.

Dans les antécédents familiaux on nota que la mère avait eu deux avortements spontanés dans le premier trimestre de la grossesse.

Cas No. 5 ( Figure 1.2)

II s'agit d'un garçon né suite à un accouchement qui s'était déroulé normalement. Cependant le liquide amniotique était brunâtre (présence de méconium indiquant une souffrance foetale); l'enfant fut réanimé.

A la naissance on nota une hypotonie sévère, des fontanelles larges, une micrognathie, un palais ogival et de l'hypertélorisme. La TACO cérébrale a mis en évidence une agénésie du corps calleux. La radiographie des genoux a montré des calcifications épiphysaires. Le diagnostic de Zellweger a été confirmé par l'étude des acides gras à longue chaîne. L'évolution de la 
maladie a été caractérisée par de l'hématémèse et des épisodes d'apnée. Le décès est survenu à 8 semaines; l'autopsie a été refusée. II a une soeur phénotypiquement normale .

Cas No. 6 ( Figure 1.2)

II s'agit d'une fille née par césarienne. Elle avait un teint grisâtre à la naissance et elle fut réanimée. L'enfant était hypotonique, avait des fontanelles larges, une micrognathie et un palais normal. Les réflexes de Moro, de succion et de préhension étaient absents. La radiographie des genoux et des têtes fémorales montra des traînées de calcifications en forme de L. La TACO cérébrale montra une atrophie cérébrale et une dilatation ventriculaire dans la région occipitale. Le décès est survenu à 2 semaines. L'autopsie révéla une maladie microkystique diffuse des reins; l'examen microscopique du foie montra une absence de peroxisomes.

Les tableaux 1.3 et $1.3 \mathrm{~A}$ résument les caractéristiques cliniques des six enfants présentant un syndrome de Zellweger au SLSJ. 
TABLEAU 1.3

Caractéristiques cliniques plus fréquemment rencontrées chez les enfants atteints du syndrome de Zellweger au SLSJ

\begin{tabular}{|c|c|c|c|c|c|c|c|}
\hline $\begin{array}{l}\text { Caractéristiques } \\
\text { cliniques }\end{array}$ & 1 & 2 & 3 & 4 & 5 & 6 & Ratio \\
\hline Fontanelles larges & + & + & + & + & + & + & $6 / 6$ \\
\hline Front proéminent & + & + & + & + & & & $4 / 6$ \\
\hline Micrognathie & + & + & + & + & + & + & $6 / 6$ \\
\hline Palais ogival & + & + & + & + & + & - & $5 / 6$ \\
\hline Hypotonie & + & + & + & + & + & + & $6 / 6$ \\
\hline Aréflexie & + & + & + & + & & + & $5 / 6$ \\
\hline Retard psychomoteur & + & + & + & + & + & + & $6 / 6$ \\
\hline Retard de croissance & + & + & + & + & + & + & $6 / 6$ \\
\hline $\begin{array}{l}\text { Réflexe de Moro absent } \\
\text { ou faible }\end{array}$ & + & + & + & + & & + & $5 / 6$ \\
\hline $\begin{array}{l}\text { Réflexe de succion } \\
\text { absent ou faible }\end{array}$ & + & + & + & + & & + & $5 / 6$ \\
\hline Convulsions & + & & + & + & + & + & $5 / 6$ \\
\hline Hépatomégalie & + & & + & + & & + & $4 / 6$ \\
\hline Caryotype normal & + & & & + & + & + & $4 / 6$ \\
\hline Age au décès & $\begin{array}{c}17 \\
\text { sem. }\end{array}$ & $\begin{array}{l}\text { Quel } \\
\text { ques } \\
\text { mois }\end{array}$ & $\begin{array}{l}\text { Quel } \\
\text { ques } \\
\text { mois }\end{array}$ & $\begin{array}{c}5 \\
\text { sem. }\end{array}$ & $\begin{array}{c}8 \\
\text { sem }\end{array}$ & $\begin{array}{c}2 \\
\text { sem. }\end{array}$ & \\
\hline
\end{tabular}


TABLEAU 1.3.A

Caractéristiques cliniques moins fréquemment rencontrées chez les enfants atteints du syndrome de Zellweger au SLSJ

\begin{tabular}{|c|c|c|c|c|c|c|c|}
\hline $\begin{array}{l}\text { Caractéristiques } \\
\text { cliniques }\end{array}$ & 1 & 2 & 3 & 4 & 5 & 6 & Ratio \\
\hline Hypertélorisme & & & & + & + & & $2 / 6$ \\
\hline $\begin{array}{l}\text { Arcades sourcillières } \\
\text { effacées }\end{array}$ & & & & + & & + & $2 / 6$ \\
\hline Epicanthus & + & + & + & & & & $3 / 6$ \\
\hline $\begin{array}{l}\text { Oreilles petites et } \\
\text { basses }\end{array}$ & & & & + & + & & $2 / 6$ \\
\hline Nystagmus & + & & & + & & & $2 / 6$ \\
\hline Opacités cornéennes & + & + & & & & + & $3 / 6$ \\
\hline $\begin{array}{l}\text { Pli palmaire transverse } \\
\text { unique }\end{array}$ & + & & & & & + & $2 / 6$ \\
\hline Ictère & + & & & + & & + & $3 / 6$ \\
\hline $\begin{array}{l}\text { Réflexe de préhention } \\
\text { absent ou faible }\end{array}$ & + & & & & & + & $2 / 6$ \\
\hline Mains en flexion & & + & & & $(+)$ & + & $3 / 6$ \\
\hline Pieds en varus équint & & & & + & & & $1 / 6$ \\
\hline Cryptorchidie & & + & & + & & & $2 / 6$ \\
\hline Splénomégalie & + & & + & & & + & $3 / 6$ \\
\hline Reins polykystiques & & & + & + & & + & $3 / 6$ \\
\hline $\begin{array}{l}\text { Naissance par césa- } \\
\text { rienne }\end{array}$ & + & - & - & - & - & + & $2 / 6$ \\
\hline $\begin{array}{l}\text { Réanimation à la nais- } \\
\text { sance }\end{array}$ & - & - & - & \pm & + & + & $3 / 6$ \\
\hline
\end{tabular}




\section{III.2 Épidémiologie et génétique}

\section{III.2.1 Incidence du syndrome de Zellweger au SLSJ}

Entre 1971 et 1990 il y a eu 95514 naissances au SLSJ. L'incidence du syndrome de Zellweger est de 1 pour 23878 naissances. II s'agit ici d'une incidence à la naissance minimum puisqu'elle a été calculée sur la base de quatre enfants seulement, car nous ne connaissions pas les dates de naissance des enfants No.2 et No.3.

Le taux de porteurs est de 1 pour 77 habitants.

III.2.2 Distribution spatiale des lieux de naissance des enfants atteints et de leurs parents

La figure 1.3 montre la distribution des lieux de naissance des enfants atteints. Quatre enfants sont nés à Chicoutimi, un à StDavid de Falardeau, et un à La Baie.

En ce qui concerne les lieux de naissance des parents, on constate qu'ils sont presque tous originaires de la région. Deux sont nés à St-Léon, deux à Chicoutimi, deux à La Baie, un à St-Stanislas et le dernier est né en dehors de la région du SLSJ. II faut rappeler ici que pour les six enfants nous observons quatre fratries, c'est pourquoi nous dénombrons seulement huit parents ( Figure 1.3). 
FIGURE 1.3 : Distribution des lieux de naissance des enfants et des porteurs obligatoires du syndrome de Zellweger au Saguenay-Lac-StJean. 


\section{LA REGION DU SAGUENAY LAC-SAINT-JEAN}

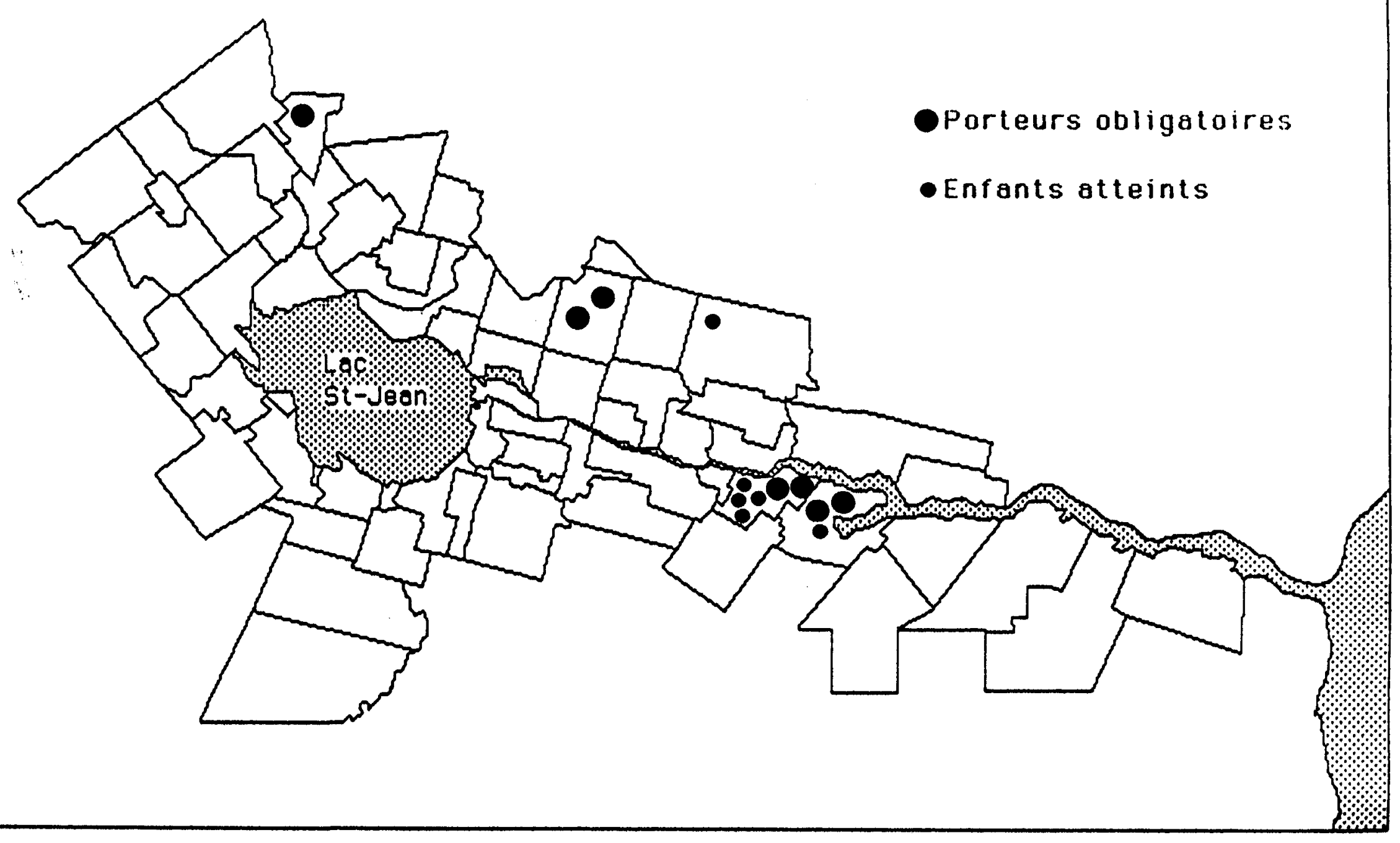




\section{III.2.3 Consanguinité et parenté}

Le coefficient moyen de consanguinité calculé pour les quatre ascendances donne une valeur égale à zéro. Aucun ancêtre commun au père et à la mère n'a été trouvé à six générations de profondeur (Tableau 1.4).

Le coefficient moyen de parenté également calculé pour les quatre ascendances, est égal à $6,61 \times 10^{-4}$. On a trouvé un ancêtre commun à la cinquième génération entre deux enfants atteints (Tableau 1.4). La moyenne des coefficients moyens de parenté des groupes-témoins est de $0,97 \times 10^{-4}$. 
TABLEAU 1.4

Coefficients moyens de consanguinité et de parenté chez les enfants atteints du syndrome de Zellweger et des groupes-témoins au SLSJ.

\begin{tabular}{|lcc|}
\hline Groupes & $\begin{array}{c}\text { Coefficient moyen } \\
\text { de consanguinité }\end{array}$ & $\begin{array}{c}\text { Coefficient moyen } \\
\text { de parenté }\end{array}$ \\
\hline $\begin{array}{l}\text { Syndrome de } \\
\text { Zellweger }\end{array}$ & 0,0 & $(\mathbf{x})-4)$ \\
Témoin 1 & 0,0 & 6,61 \\
Témoin 2 & 0,0 & 0,0 \\
Témoin 3 & 0,0 & 0,1 \\
\hline
\end{tabular}


CHAPITRE IV

DISCUSSION

$\uparrow$ 
Bien que le syndrome de Zellweger ne soit pas connu de la population du Saguenay-lac-St-Jean, il n'en demeure pas moins que la concentration d'enfants atteints dans cette région est importante par rapport à ce qu'on devrait s'attendre étant donné la taille de la population. En effet la population du SLSJ compte près de 300000 habitants. L'incidence du syndrome de Zellweger est de 1 cas pour 23 878 naissances comparativement aux Etats-unis où l'incidence a été calculée par le Kennedy Institute, à 0.79 cas pour 100000 naissances, soit 1 cas pour 127000 naissances (Lazarow et Moser 1989). Comme on peut le constater l'incidence du syndrome de Zellweger au SLSJ est de quatre à cinq fois plus élevée qu'au Kennedy Institute. II est important de rappeler ici que l'incidence calculée pour le SLSJ est une incidence minimum (sur la base de quatre enfants atteints). II n'est pas impossible qu'il y ait eu d'autres naissances d'enfants atteints dans la région du Lac-St-Jean qui ne seraient pas connues des pédiatres du SLSJ. En effet, les enfants atteints connus sont tous nés au Saguenay; on peut supposer que d'autres cas ont pu être rencontrés au Lac-St-Jean. Étant donné le faible échantillonnage de cette population, l'incidence du syndrome de Zellweger pourrait être aussi basse que 1/1.200.000 ou aussi élevée que $1 / 12.000$ (intervalle de confiance à 95\%). Étant donné qu'il est impossible de déterminer l'intervalle de confiance des incidences rapportées dans la littérature, aucune comparaison n'est possible. 
Le taux de porteurs au SLSJ est de 1 pour 77 habitants comparativement à 1 pour 178 habitants au Kennedy Institute: Le taux de porteurs au SLSJ est donc 2,3 fois plus élevé.

Les phénotypes des enfants atteints de cette maladie au SLSJ sont très semblables pour ne pas dire identiques à ceux décrits dans la littérature. Si l'on regarde le tableau 1.5, on peut voir que la plupart des caractéristiques décrites dans la littérature sont présentes chez les enfants du SLSJ et cela même si le nombre de cas étudiés au SLSJ est très restreint. D'autres caractéristiques auraient pu se rajouter au tableau clinique des enfants du SLSJ mais l'interprétation de certaines données prêtait à confusion, comme par exemple l'expression "tête carrée" qui a été rencontrée mais fut par la suite écartée de l'étude car trop imprécise.

Des six enfants étudiés, trois faisaient partie de la même fratrie diminuant à quatre le nombre de familles et diminuant par le fait même le nombre de généalogies à reconstituer. Etant donné ce nombre restreint de familles il ne nous était pas possible de réaliser une étude statistique de la distribution des lieux de naissance des parents et des enfants atteints, par contre il était important de constater que tous les parents sauf un ( $7 / 8$ ) sont originaires de la région du SLSJ.

Aucune consanguinité proche n'a été observée dans ce groupe cible, ce qui semble confirmer la fréquence élevée du gène dans la population. Par contre le coefficient moyen de parenté est 6,8 fois plus élevé que dans les groupes témoins. Cela suggère que c'est la même mutation que l'on retrouve chez tous les enfants atteints de la région. 
TABLEAU 1.5

Comparaison entre les caractéristiques trouvées dans la littérature et celles des patients du SLSJ

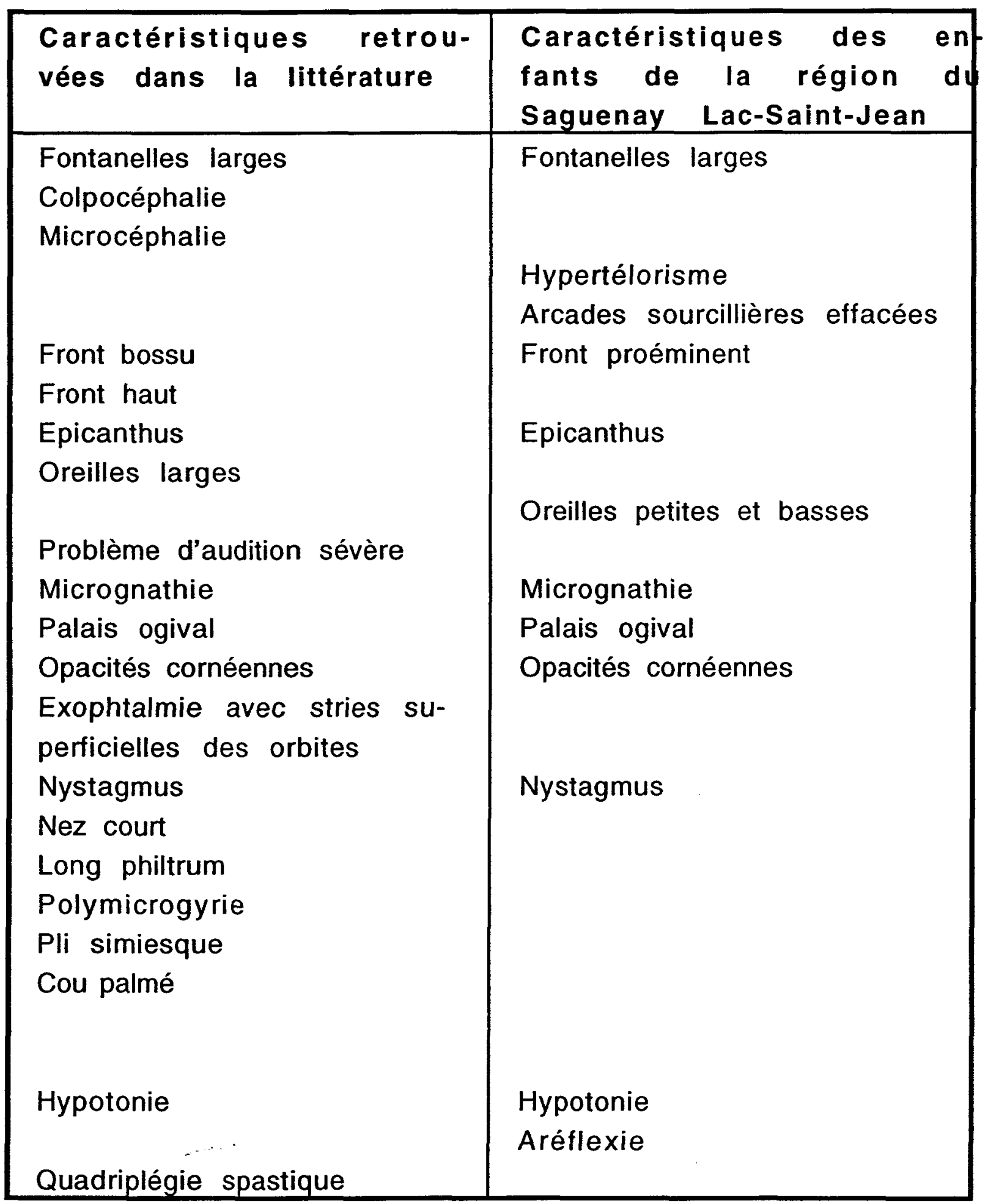


TABLEAU 1.5 (suite)

Retard psychomoteur

Retard de croissance pré- et post-natal

Réflexes de succion faibles
Retard psychomoteur

Retard de croissance

Réflexes de succion absents ou faibles

Réflexe de préhention absent ou faible

Réflexes de Moro absents

Cyphoscoliose

Contractures aux articulations

Calcifications des rotules

Calcifications des omoplates

Calcifications des vertèbres

Pieds en varus équin

Déviation ulnaire de la main

Ictère

Hépatomégalie

Hernie inguinale

Sténose pulmonaire

Reins polykystiques

Hypogonadisme

Cryptorchidie

Convulsions
Pieds en varus équin

Mains en flexion

Pli palmaire transverse unique

Ictère

Hépatomégalie

Splénomégalie

Reins polykystiques

Cryptorchidie

Convulsions 


\section{CONCLUSION}

Le fait de retrouver au SLSJ un tableau clinique comparable entre les enfants atteints du syndrome de Zellweger, le fait que la maladie semble avoir une incidence plus élevée que dans la littérature, le fait aussi qu'il y ait absence de consanguinité proche mais un coefficient de parenté élevé et que les lieux de naissance des parents soient presque tous situés à l'intérieur de la région, tout cela contribue à suggérer qu'on a affaire à un gène fréquent dans cette population.

Bien que l'incidence du syndrome de Zellweger soit élevée dans la région comparativement à l'incidence mondiale, on ne peut pas considérer que cette maladie soit très répandue dans la région. En fait, l'incidence du syndrome de Zellweger n'atteint pas un niveau comparable à d'autres maladies à transmission autosomale récessive comme la fibrose kystique ou la tyrosinémie qui sont beaucoup plus répandues dans la région (De Braekeleer 1991).

Le syndrome de Zellweger reste quand même une maladie létale et peu connue. L'espoir de trouver un traitement efficace n'est pas à envisager pour le moment, il ne reste que le dépistage prénatal à envisager pour les personnes dont un cas s'est déjà déclaré dans la famille. 


\section{BIBLIOGRAPHIE}

Adelbert A.D., Hoefler S., Hoefler G., Paschke E., Paltauf F., Moser A., Moser H. (1989) Genetic and phenotypic heterogeneity in disorders of peroxisome biogenesis : A complementation study involving cell lines from 19 patients. Pediatr Res 26: 67-72.

Agamanolis D.P., Robinson H.B., Timmons G.D. (1976) Cerebro-hepatorenal syndrome. Report of a case with histochemical and ultrastructural observations. J Neuropathol Exp Neurol 35: 226-246.

Ahlabo I., Barnard T. (1971) Observations on peroxisomes in brown adipose tissue of the rat. J Histochem Cytochem 19: 670-675.

Bjorkheim I., Blomstrand S., Glaumann H., Strandvik B. (1985) Unsuccessful attempts to induce peroxisomes in two cases of Zellweger disease by treatment with clofibrate. Pediatr Res19: 590593.

Bouchard G., De Braekeleer M. (1991) Histoire d'un Génôme. Presses de l'Université du Québec, Sillery.

Cohen S.M.Z., Brown F.R., Martyn L., Moser H.W., Chen W., Kisten-Macher M., Punett H., Grover W., De Al Cruz C., Chan N.R., Green W.R. (1984) Ocular histopathological and biochemical studies of the cerebrohepato-renal (Zellweger) syndrome and its relation to neonatal adrenoleukodystrophy. Am J Ophtalmol 96: 488-501.

De Braekeleer M. (1991) Hereditary disorders in Saguenay-Lac-StJean (Québec, Canada). Hum Hered 41: 141-146.

De Braekeleer M., Larochelle J. (1990) Genetic epidemiology of hereditary tyrosinemia in Quebec and in Saguenay-Lac-St-Jean. Am J Hum Genet 47: 302-307.

De Duve C., Baudhuin P. (1966) Peroxisomes (microbodies and related particles). Physiol Rev 46: 3230-357. 
Deleon G.A., Grover W.D., Huff D.S., Morinigo-Mestre G., Punnett H.P., Kistenmacher M.L. (1977) Globoid cells, glial nodules, and peculiar fibrillary changes in the cerebro-hepato-renal syndrome of Zellweger. Ann Neurol 2: 473-484.

Evrard P., Caviness V.S., Prats-Vinas J., Lyon G. (1978) The mechanism of arrest of neuronal migration in the Zellweger malformation : An hypothesis based upon cytoarchitectonic analysis. Acta Neuropathol (Berlin) 41: 109-119.

Gilchrist K.W. , Gilbert E.F., Goldfarb S., Goll U., Spranger J.W., Opitz J.M. (1976) Studies of malformation syndromes of man XIB: The cérébro-hepato-renal syndrome of Zellweger: Comparative pathology. Eur J Pediatr 121: 99-118.

Golden N.W., Holmes R.G., Custer J., Lipkowitz J.L., Stover J., Datta N., Hajra A. (1986) Zellweger syndrome: Diagnostic assays, syndrome delineation, and potential therapy. Am J Med Genet 24: 69-82.

Govaerts L., Monnens L., Tegelaers W., Trijbels F., van Ray-Selten A. (1982) Cerebro-hepato-renal syndrome of Zellweger: Clinical symptoms and relevant laboratory findings in 16 patients. Eur $\mathrm{J}$ Pediatr 139: 125-128.

Hajra A.J., Datta N.S., Jackson L.G., Moser A.B., Moser H.W., Larsen J.W., Powers J. (1985) Prenatal diagnosis of Zellweger cerebro-hepatorenal syndrome. $\mathrm{N}$ Engl J Med 312: 445-446.

Heymans H.S.A. (1984) Cerebro-hepato-renal syndrome (Zellweger) syndrome. Clinical and biochemical consequences of peroxisomal dysfunction. Thesis, University of Amsterdam.

Holmes R.D., Wilson G.N., Hajra A.K. (1987) Oral ether lipid therapy in patients with peroxisomal disorders. J Inherit Metab Dis 10: 239241.

Hruban Z., Rechigl M. (1969) Microbodies and related particles. Academic Press, New York.

Hugo M., Moser A.B. (1990) Adrenoleukodystrophy and Zellweger syndrome. Prog Clin Biol Res 321: 511-535. 
Jacquard A. (1974) Génétique des populations humaines. Presses Universitaires de France, Paris.

Lazarow P.B., Moser H.W. (1989) Disorders of peroxisome biogenesis. In: Scriver C.R., Beaudet A.L., Sly W.S., Valle D. (eds.). The metabolic basis of inherited diseases. New York, McGraw Hill, pp. 1479-1509.

Lazarow P.B., Small G.M., Santos M., Shio H., Moser A., Moser H., Esterman A., Black V., Dancis J. (1988) Zellweger syndrome amniocytes: Morphological appearance and a simple sedimentation method for prenatal diagnosis. Pediatr Res 24: 63-67.

Lazarow P.B., Shio H., Robbi M. (1980) Biogenesis of peroxisomes and the peroxisome reticulum hypothesis. In: Bucher T., Sebald W., Weiss $H$. (eds.). Biological chemistry of organelle formation. New York, Springer-Verlag, pp. 187-207.

Lazarow P.B., Black V., Shio H., Fujiki Y., Hajra A.K., Datta N.S., Bangaru B.S., Dancis J. (1985) Zellweger syndrome: Biochemical and morphological studies on two patients treated with clofibrate. Pediatr Res 19: 1356-1364.

Moser A.B., Borel J., Odone A., Naidu S., Cornblath D., Sanders D.B., Moser H.W. (1987) A new dietary therapy for adrenoleukodystrophy. Biochemical and preliminary clinical results in 36 patients. Ann Neurol 21: 240-247.

Moser A.E., Singh I., Brown F.R., Solish G.I., Kelley R.I., Benke P.J., Moser H.W. (1984) The cerebro-hepato-renal (Zellweger) syndrome: Increased levels and impaired degradation of very long chain fatty acids and their use in prenatal diagnosis. N Engl $\mathrm{J}$ Med 310 : 11411146.

Naritomi K., Hyakuna N., Suzuki Y., Orii T., Hirayama K. (1988) Zellweger syndrome and a microdeletion of the proximal long arm of chromosome 7. Hum Genet 80: 201-202.

Opitz J.M., Zurheim B.M., Vitale L., Gerritsen N.T. (1969) The Zellweger syndrome (cerebro-hepato-renal syndrome. In: Bergsma D. (ed.). Malformation syndromes, Part II. Baltimore, Williams and Wilkins for the National Foundation - March of Dimes BD 2: 144-158. 
Passarge E., McAdams A.J. (1967) Cerebro-hepato-renal syndrome. A newly recognized hereditary disorder of multiple congenital defects including sudanophilic leukodystrophy, cirrhosis of the liver, and polycystic kidneys. J Pediatr 71: 691-702.

Powers, J.M., Moser H.W., Moser A.B., Upshur J.K., Bradford B.F., Pai S.G., Kohn P.H., Frais J., Tiffany C.(1985) Fetal cerebro-hepato-renal (Zellweger) syndrome : Dysmorphic, radiologic, biological pathologic findings in four attected fetuses. Hum Pathol 16: 610-620.

Powers J.M., Tummons R.C., Moser A.B., Moser H.W., Huff D.S., Kelley R.I. (1987) Neuronal lipidosis and neuroaxonal dystrophy in cerebrohepato-renal (Zellweger) syndrome. Acta Neuropathol 73: 333-343.

Rocchiccioli F., Aubourg P., Choiset A. (1987) Immediate prenatal diagnosis of Zellweger syndrome by direct measurement of very long chain fatty acids in chorionic villus cells. Prenat Diagn 7: 349-354.

Roels F., Verdonck V., Pauwels M., Foulon W., Lissens W., Liebaers I. (1987) Visualization of peroxisomes and plasmalogens in first trimester chorionic villus. J Inherit Metab Dis 10: 223-235.

Schutgens R.B.H., Schrakamp G., Wanders R.J.A., Heymans H.S.A., Moser H.W., Moser A.E., Tager J.M., Van den Bosch H., Aubourg P. (1985) The cerebro-hepato-renal (Zellweger) syndrome : Prenatal detection based on impaired biosynthesis of plasmalogens. Prenat Diagn 5: 337344 .

Schutgens R.B.H., Heymans H.S.A., Wanders R.J.A., Van den Bosch H., Tager J.M. (1986) Peroxisomal disorders: A newly recognised group of genetic diseases. Eur J Pediatr 144: 430-440.

Tager J.M., Brul S., Wiemer E.A.C., Strijland A., Driel R.V., Schutgens R.B.H., Van den Bosch H., Wanders R.J.A., Westerveld A. (1990) Genetic relationship between the Zellweger syndrome and other peroxisomal disorders characterized by an impairment in the assembly of peroxisomes. Prog Clin Biol Res 321: 545-558. 
Versmold, H.T., Bremer H.J., Herzog V., Siegel G., Bassewitz D.B.V., Irle U., Voss H.V., Lombeck I., Brauser B. (1977) A metabolic disorder similar to Zellweger syndrome with hepatic acatalasia and absence of peroxisomes, altered content and redox state of cytochrome, and infantile cirrhosis with hemosiderosis. Eur J Pediatr 124: 261-275.

Vitale L., Opitz J.M., Shahidi N.T. (1969) Congenital and familial iron overload. N Engl J Med 280: 642-645.

Wanders R.J.A., Van Wijland M.J.A., Van Roermund C.W.T., Schutgens R.B.H., Van den Bosch H., Tager J.M., Nijenhuis A., Tromp A. (1987) Prenatal diagnosis of Zellweger syndrome by measurement of very long chain fatty acid (C26:0) beta oxidation in cultured chorionic villous fibroblasts: Implications for early diagnosis of other peroxisomal disorders. Clin Chim Acta 165: 303-310.

Wilson G.N., Holmes R.G., Custer J., Lipkowitz J.L., Stover J., Datta N., Hajra A. (1986). Zellweger syndrome : Diagnostic assays, syndrome delineation, and potential therapy. Am J Med Genet 24: 69-82. 


\section{DEUXIEME PARTIE}

ÉTUDE CLINIQUE, GÉNÉTIQUE ET ÉPIDÉMIOLOGIQUE DE LA CYSTINOSE AU SAGUENAY-LAC-ST-JEAN. 
CHAPITRE I

REVUE DE LA LITTÉRATURE 


\section{I.1 Aspects cliniques et historiques de la cystinose}

\section{I.1.1 Historique}

La première description de la cystinose a été faite en 1903 par Emil Abderhalden. Le propositus était un garçon âgé de 21 mois et demi, mort d'inanition. A l'autopsie il remarqua que ses organes avaient un aspect blanchâtre et présentaient des lésions. L'analyse chimique montra l'accumulation d'un élément soit l'acide aminé cystine (Abderhalden,1903). Dans les années 1930 on identifia la cystinose comme une cause de glycosurie non diabétique, on nota également une association avec le rachitisme rénal (syndrome de Fanconi) et un retard staturo-pondéral (Fanconi, 1936). Mais ce n'est qu'en 1958 que Worthen et Good firent la différence entre le syndrome de Fanconi et la cystinose.

En 1967 les investigations modernes de la cystinose commencèrent lorsque la nature intracellulaire de l'emmagasinage de la cystine fut défini (Gahl et al, 1989).

\subsubsection{Principales caractéristiques cliniques}

La cystinose est une maladie autosomale récessive rare. Elle est due à l'accumulation de l'acide aminé cystine dans les lysosomes de tous les organes du corps, les endommageant par ce fait même à différents degrés. 
Les enfants atteints de cystinose néphropathique sont normaux à la naissance à l'exception de la pigmentation de leur peau et de leurs cheveux qui est nettement plus claire que leurs frères et soeurs. Mais vers l'âge de six mois généralement, les bébés atteints peuvent présenter un retard staturo-pondéral, ils mangent très peu, sont accaparants, urinent et boivent excessivement et développent ce qu'on appelle le syndrome de Toni Debré Fanconi (tubulopathie rénale). Ce syndrome entraîne de la déshydratation, des périodes récurrentes d'acidose, des vomissements, des déséquilibres électrolytiques, de l'hypophosphatémie, du rachitisme et un retard staturo-pondéral. La circonférence de la tête est normale et l'intelligence est épargnée. Les autres manifestations de la cystinose sont: la photophobie, l'hypothyroïdie et une diminution de la sudation (Gahl et al, 1989).

Le dommage causé au glomérule du rein progresse inexorablement et le patient doit être mis sous dialyse habituellement entre l'âge de six et douze ans. La transplantation est requise et lorsqu'elle est pratiquée, l'accumulation de cystine n'affecte pas le nouveau rein mais continue de s'accumuler dans les autres organes. Les transplantés souffrent donc de désordres comme l'érosion de la cornée, de cécité, de diabète sucré et dans une bonne proportion de détérioration neurologique (Gahl et al, 1989).

$\mathrm{Au}$ point de vue clinique, les cas de cystinose varient beaucoup de l'un à l'autre, en passant du cas bénin de cystinose d'un adulte avec des cristaux de cystine dans la cornée mais n'ayant pas de maladie de rein, au cas intermédiaire de cystinose de l'adolescent caractérisé par une détérioration lente des reins et finalement au cas classique de cystinose des reins de l'enfant; cette forme de cystinose 
est létale si une transplantation rénale n'est pas pratiquée. Les hétérozygotes sont phénotypiquement normaux (Gahl et al, 1989).

\section{I.1.3 Manifestations oculaires de la cystinose}

Dans la première année de vie la présence de cristaux de cystine dans la cornée ne peut être détectée par l'examen à la lampe à fente. Par la suite les cristaux se retrouvent généralement dans la partie périphérique et antérieure de la cornée. Chez les patients plus âgés les cristaux de cystine se distribuent d'un bout à l'autre du stroma cornéen. Ils peuvent être également présents dans la conjonctive et dans l'iris. Par contre un groupe de chercheurs a montré que chez les patients d'origine canadienne-française, il y a une absence de dépôts de cristaux de cystine dans l'épithélium de la cornée; en fait l'atteinte ophtalmique en général est très légère chez ce groupe (Richler et al.1991).

Les cristaux de cystine n'ont pas la même apparence selon l'endroit où ils se situent; ceux situés dans la cornée ont l'aspect d'une "myriade de petites aiguilles réfringentes" (Richler et al.1991), et ceux présents dans la conjonctive sont plutôt d'apparence blanchâtre.

Chez tous les patients l'examen du fond d'oeil est anormal. Les patients plus jeunes ne présentent qu'une très légère marbrure de l'épithélium pigmentaire au niveau de la rétine. Par contre les patients plus âgés présentent un pigment trouble et une zone de dépigmentation de la rétine (Richler et al.1991). 
Les fonctions comme l'acuité, la reconnaissance des couleurs et la vision de nuit sont normales chez la plupart des jeunes patients atteints de cystinose. Mais depuis que des transplantations sont pratiquées les problèmes reliés à la vision s'avèrent plus importants dans ce groupe. En effet, ces patients souffrent d'une diminution de la faculté à reconnaître les couleurs ainsi que d'une plus grande difficulté à s'adapter à la noirceur; l'acuité visuelle diminue par la suite (Gahl et al, 1989).

Les autres complications oculaires dues à la cystinose sont: la photophobie, un blépharospasme, un épaississement de l'iris qui peut résulter en un glaucome à cause des dépôts de cystine dans les segments antérieurs, et plus rarement, une dégénérescence de la cornée qui, combinée à une kératopathie peut mener à une ulcération de la cornée (Richler et al.1991).

La rétinopathie associée à la cystinose est irréversible, seul le traitement des complications au niveau de la cornée, est possible. La cystéamine a déjà été utilisée en application topique sur les yeux, mais ce traitement s'avéra décevant car il y avait plus de désagréments que d'amélioration (Richler et al.1991).

La dégénérescence oculaire est très débilitante pour les patients car elle leur nuit dans la pratique d'activités courantes comme la conduite d'une automobile, l'attention scolaire et le travail (Gahl et al.1988). 


\section{I.1.4 Croissance}

Bien que les enfants atteints de cystinose aient un poids et une taille normale à la naissance, leur courbe de croissance commence à décrocher de leur percentile dès la première année de vie et cela d'une façon si rapide qu'à la fin de la seconde année ils sont presque nains. Le retard de croissance est très sévère car les enfants peuvent perdre jusqu'à trois percentiles par année d'âge (Gahl et al. 1989).

De toutes les maladies comportant une atteinte rénale, la cystinose est celle qui affecte le plus la croissance. Ce retard de croissance peut évidemment être le résultat d'une acidose chronique, d'une insuffisance et d'un rachitisme rénal mais un autre facteur viendrait amplifier ce phénomène. En effet, cette hypothèse serait que l'accumulation de cystine dans les autres organes comme dans les os longs du corps affecte la croissance en empêchant les cellules de se répliquer. Le fait que les enfants traités à la cystéamine aient une croissance plus normale vient renforcer cette hypothèse (Gahl et al, 1989).

La croissance chez les patients qui ont reçu une transplantation varie d'une personne à l'autre, mais plus la transplantation est pratiquée tôt, plus la croissance a des chances de se dérouler normalement.

La maturation sexuelle après une transplantation semble retardée chez les garçons. La puberté chez les garçons s'effectue en moyenne vers l'âge de 16 à 17 ans. Plusieurs présentent un 
hypogonadisme et ont besoin de testostérone. L'accumulation de cystine dans les testicules détruit ce parenchyme. La fécondité des hommes atteints de cystinose est douteuse car aucun homme atteint adulte n'a été rapporté comme étant père d'un enfant. Toutefois une femme atteinte de cystinose âgée de 20 ans a mis au monde un fils. Enfin, la puberté chez les filles s'effectue environ vers 14 ou 15 ans (Gahl et al.1988).

\subsection{Diagnostic}

Depuis les années 70, l'âge moyen pour le diagnostic de cystinose (pour les patients encore vivants en 1985) était approximativement de 28 mois, mais aujourd'hui cette moyenne a probablement été diminuée (Gahl et al. 1986).

La méthode la plus directe est de mesurer la quantité de cystine contenue dans les leucocytes. En effet, la quantité de cystine contenue dans les leucocytes des homozygotes est de 50 à 100 fois supérieure à celle d'un individu normal. Cette méthode offre plusieurs avantages soit d'être peu traumatisante pour le patient, très rapide et concluante (Schneider et al. 1967; Gahl et al. 1984).

Des cultures de fibroblastes de la peau des personnes atteintes sont aussi assez souvent utilisées pour le diagnostic de cystinose. La quantité de cystine mesurée dans ces cultures varie entre 6 et 14 fois le niveau normal (Schneider et al. 1967; Schneider et al. 1968; Goldman et al. 1971).

La cystinose peut être diagnostiquée par un examen ophtalmologique révélant des dépôts de cristaux de cystine au niveau 
de la cornée chez les patients de plus d'un an. Cet examen rapide et facile se pratique à l'aide de la lampe à fente (Wong, 1973).

II existe d'autres méthodes pour la détection des cristaux de cystine comme par exemple la spectroscopie à infra-rouges des cheveux d'une personne atteinte (Lubec et al. 1983), et une biopsie de la conjonctive ou de la muqueuse rectale (Cogan et Kuwabara, 1960; Holtzapple et al. 1969).

Le diagnostic des hétérozygotes peut s'effectuer à partir des dépôts de cystine contenus dans les leucocytes ou dans les cultures de fibroblastes. Le niveau de cystine contenu dans les leucocytes n'est pas toujours différent de celui observé chez les individus normaux. Par contre le niveau de cystine contenu dans les cultures de fibroblastes se situe à un niveau légèrement supérieur à la moyenne qui est de $0,2 \mathrm{mmol}$ par milligramme de protéine, mais les hétérozygotes emmagasinent moins de $1.0 \mathrm{mmol}$ par milligramme de protéine (Schneider et al. 1967; Gahl et al. 1984).

Le diagnostic prénatal s'effectue à l'aide de l'analyse du liquide amniotique entre 14 et 18 semaines de gestation (Schneider et al 1974; States et al.1975; Boman et Schneider, 1981). Récemment l'étude des cellules chorioniques a montré un haut taux de cystine à seulement 9 semaines de gestation (Gahl et al. 1985).

\subsection{Traitement}

Les enfants atteints de cystinose développent comme il a été dit précédemment, un syndrome de Fanconi tôt dans l'enfance, ce qui a comme conséquence des pertes excessives de glucose, d'eau, de 
sodium, de potassium, de bicarbonate, de phosphore et de carnitine (Gahl et al, 1989). Des suppléments oraux d'électrolytes faits à partir d'une combinaison de sodium, de potassium et de bicarbonates sont prescrits pour combler ces déséquilibres. Lorsque des périodes de gastro-entérites surviennent, de fortes doses d'électrolytes sont requises par perfusion intraveineuse (Gahl et al. 1989).

En cas d'insuffisance rénale la dialyse est requise et la seule solution envisageable à long terme est la transplantation.

Plusieurs substances ont été utilisées pour éliminer la cystine dans les cellules comme l'acide ascorbique, le dithiothreitol et la pantéthine; ces produits se sont montrés efficaces pour l'élimination de la cystine lysosomale mais sans aucune amélioration de la croissance et des fonctions rénales (Gahl et al. 1987).

Le traitement à la cystéamine est pour le moment le plus efficace (le mode d'action de la cystéamine sera expliqué plus loin dans la partie 1.4 "Défaut biochimique"). En effet la fonction glomérulaire du rein est entièrement normale chez les patients de plus de dix ans traités à la cystéamine. Cela laisse supposer que la cystéamine peut prévenir indéfiniment l'urémie chez des patients traités tôt dans l'enfance. La cystéamine présente comme avantage aussi de rétablir la croissance des enfants atteints en éliminant la somatostatine, une hormone inhibitrice des hormones de croissance (Gahl et al. 1987).

Les chercheurs n'ont pas remarqué de changement pour la quantité de cristaux de cystine au niveau de la cornée des enfants atteints. Par contre pour les transplantés la cystéamine peut aider à conserver intactes les fonctions des autres organes (Gahl et al.1987). 
Les principaux inconvénients de la cystéamine sont sa mauvaise odeur et son goût désagréable déplorés par les enfants atteints et leurs parents. Mais maintenant des compagnies pharmaceutiques ont mis au point un autre agent soit la phosphocystéamine qui n'a pas ces inconvénients (Gahl et al.1987).

\subsection{Défaut biochimique}

Une première hypothèse pour expliquer l'emmagasinage de la cystine dans la cystinose est que le mécanisme de transport des acides aminés à travers la membrane des lysosomes, est défectueux dans les cellules mutantes. Par la suite ce concept gagna plus de crédibilité par l'appui d'autres études portant sur les vacuoles des macrophages des souris. En effet ces études démontrèrent que lorsque les peptides sont de poids moléculaire supérieur à 220 ou 230 daltons, les lysosomes cellulaires commencent à former une vacuole présumément parce que ces peptides ne peuvent pas traverser librement la membrane du lysosome, ils ont donc tendance à s'accumuler ( Ehrenreich et Cohn, 1969).

Dans des expériences subséquentes la caractéristique de perméabilité de la membrane des lysosomes fut explorée. On démontra que dans les fibroblastes des personnes atteintes de cystinose, les lysosomes forment des vacuoles lorsqu'il sont exposés à de grandes concentrations de L-cystéine-D-pénicillamine avec un pont disulfure, et la cystine se retrouve coincée à l'intérieur du lysosome, contrairement à ce qui se passe chez les personnes normales (Schulman et Bradley, 1970). 
Le plus étudié des agents éliminant la cystine à ce jour est la cystéamine, un aminothiol qui par ses propriétés, aide à réduire le contenu en cystine des fibroblastes des personnes atteintes (Thoene et al.1976). La cystéamine réagit avec la cystine pour former la cystéine et la cystéine-cystéamine avec un pont disulfure; la cystéine est laissée libre dans les lysosomes, et la cystéinecystéamine diffuse hors du lysosome parce que son poids moléculaire est inférieur à 220 daltons, qui est la limite supérieure pour le libre mouvement des acides aminés et des peptides à travers la membrane des lysosomes (Ehrenreich et Cohn, 1969).

D'autres travaux ont par la suite démontré que la cystéinecystéamine avec un pont disulfure ressemble à la structure de la lysine, et comme les personnes atteintes de cystinose ont un système de transport lysosomal intact pour la lysine, la cystine-cystéamine est transportée au travers la membrane lysosomale par le transporteur de la lysine (Pisoni et al, 1985).

La concentration en cystine dans le plasma est normale; l'absorption de cystine par l'intestin est normale aussi et le niveau de concentration dans l'urine n'est pas plus élevée que pour les autres acides aminés. Or l'accumulation de cystine chez les patients atteints de cystinose est un phénomène intracellulaire. Des cristaux ont été identifiés dans plusieurs tissus comme les reins, le foie, les poumons, le pancréas, l'intestin, l'appendice, la rate, la conjonctive, la cornée, les ganglions lymphatiques, la moelle osseuse, la thyroïde, le thymus, le plexus choroide, les leucocytes polynucléaires et les monocytes. La forme des cristaux retrouvés dans ces tissus peut être rectangulaire ou hexagonale (Gahl et al,1989). 
Certains tissus chez les personnes atteintes semblent dépourvus de cristaux; c'est le cas du cerveau, des muscles et d'une variété de leucocytes périphériques (Schulman et al. 1970); le taux d'accumulation de cystine dans ces tissus est de 5 à 500 fois supérieur au taux normal. Les tissus contenant des cristaux comme les reins, la conjonctive et le foie, ont jusqu'à 100 fois le taux de cystine des tissus n'en contenant pas, ce qui veut dire de 500 à 5000 fois le taux normal de cystine (Gahl et al, 1989).

\section{5 Épidémiologie et génétique}

La cystinose est une maladie autosomale récessive et son incidence mondiale est estimée à environ 1 pour 100000 à 1 pour 200000 naissances vivantes. Le taux de porteurs est d'environ 1 pour 200 habitants dans la population en général (Gahl et al,1989).

Cette incidence est à peu près égale dans tous les pays du monde sauf pour une province de France soit la Bretagne où l'incidence est de 1 pour 25909 naissances et la fréquence du gène est de 0.0062 , comparativement au reste de la France où l'incidence est de 1 pour 326444 naissances et la fréquence du gène est de 0.0018 (Bois et al.1976). La région du Saguenay-Lac-St-Jean est également connue pour sa concentration d'enfants atteints de cystinose mais aucune incidence n'a été calculée jusqu'à ce jour (Bergeron,1974, communication personnelle à Bois et al.1976).

II semblerait que le ratio garçons/filles soit inégal, en effet des chercheurs allemands ont remarqué que leur ratio garçons/filles était de 58:43 (Manz et Gretz,1985). Une autre étude 
en Europe a montré qu'il y avait une prépondérance en faveur des garçons soit 115:90 (Gretz, Manz et Augustin,1982) et pour les patients traités au National Institute of Child and Health and Human Development au Maryland, on observe un ratio de 31: 27 (Gahl et al, 1989).

Aucun gène spécifique pour la cystinose n'a été identifié, et aucun linkage avec des marqueurs HLA n'a été trouvé non plus (Steinherz et al, 1981). II y a peut-être plus d'un gène ou différentes mutations qui peuvent être impliqués dans cette maladie étant donné qu'il y a une grande hétérogénéité des phénotypes rencontrés. La complexité du processus de transport lysosomal n'est pas négligeable non plus et peut-être que différentes mutations produisent le même effet au point de vue biochimique, c'est-à-dire l'accumulation de cystine dans les lysosomes (Gahl et al,1989).

Une étude sur les familles issues de différentes régions de France ayant au moins un enfant atteint de cystinose a montré que les coefficients de consanguinité et de parenté de ces familles étaient plus élevés que ceux des groupes-témoins. En effet des 42 familles étudiées 6 étaient apparentées et le coefficient moyen de parenté des parents était de $53 \times 10^{-4}$ (Bois et al.1976).

Dans cette même étude les chercheurs ont regardé les lieux de naissance des parents et ils ont remarqué que le pourcentage des parents originaires des mêmes provinces était supérieur à celui des groupes-témoins, il en va de l'ordre de $79,41 \%$ contre $68 \%$. Ce pourcentage est cependant augmenté par la province de Bretagne qui à elle seule obtient un taux de $90.91 \%$ de parents originaires de cette région (Bois et al.1976). 
Ils ont également fait une étude sur les groupes sanguins des enfants atteints et ils ont constaté que $65 \%$ de ces patients appartenaient au groupe $A$. Ce phénomène reste inexpliqué puisque dans la population française on ne retrouve pas une fréquence excessif du groupe sanguin $A$, et aucune autre étude n'a été faite sur le sujet (Bois et al.1976).

Puisque la concentration des cas de cystinose en Bretagne n'est pas en corrélation avec la densité de sa population, les chercheurs s'expliquent mal cette situation. Un coefficient de consanguinité élevé ne peut à lui seul en être la cause. Les chercheurs suggèrent donc que des gènes délétères ont été conservés à une haute fréquence par une dilution imparfaite dans cette population (Bois et al.1976). 
CHAPITRE II

MATÉRIEL ET MÉTHODES 


\section{II.1 Matériel}

\section{II.1.1 Population à l'étude}

Au cours des vingt dernières années plusieurs enfants atteints de cystinose ont été traités à la Clinique de Pédiatrie de Chicoutimi. Connaissant l'incidence mondiale de cette maladie les pédiatres ont constaté que le nombre de cas rencontrés semblait plus élevé au Saguenay-Lac-St-Jean. Le docteur Jean Larochelle a contacté le docteur Marc De Braekeleer pour l'informer de ce fait. II lui a ensuite fourni la liste des enfants atteints et, après avoir obtenu l'autorisation du directeur des services professionnels de l'hôpital de Chicoutimi et de l'ensemble des pédiatres de la Clinique de Pédiatrie, les dossiers ont pu être consultés à la Clinique de Pédiatrie ainsi qu'aux archives de l'hôpital de Chicoutimi. II faut signaler qu'une enquête des pédiatres de Chicoutimi auprès de leurs confrères de la région du SLSJ n'a pas révélé d'autres cas de cystinose.

\section{II.2 Méthodologie}

\section{II.2.1 Étude clinique}

Les données qui ont été extraites des dossiers sont: la date et le lieu de naissance des patients, le lieu de résidence des parents, 
le poids et la taille à la naissance, la description des principales caractéristiques cliniques, les différents tests sanguins et urinaires, les hospitalisations, l'évolution de la maladie, la date du début du traitement à la cystéamine et la date de décès lorsqu'indiqué.

\section{II.2.2 Étude génétique et épidémiologique}

On a utilisé une méthode semblable à celle utilisée pour d'autres maladies récessives au SLSJ; cette méthodologie est expliquée plus en détails dans De Braekeleer et Larochelle (1990).

L'incidence à la naissance a été calculée pour la période 1971-1990 en divisant le nombre de naissances d'enfants atteints nés au SLSJ par le nombre total de naissances dans la région pour la même période. Le taux de porteurs a été estimé en utilisant la loi d'Hardy Weinberg (De Braekeleer et Larochelle 1990).

Les lieux de résidence des grands-parents des enfants atteints (au moment de la naissance des parents des enfants atteints) ont été extraits du fichier de population du SLSJ à SOREP.

Les généalogies ont été reconstituées automatiquement à l'aide de MEDIC 4; le coefficient de consanguinité a été calculé par le programme CONB et le coefficient de parenté a été calculé grâce au programme APPB (De Braekeleer et Larochelle 1990). Ces reconstitutions ont été faites dans le but également de trouver des fondateurs et des ancêtres communs aux porteurs obligatoires; à partir de ces données la recherche d'un effet fondateur qui se définit comme étant la formation d'une nouvelle population à partir de 
quelques individus porteurs d'un échantillon de gènes issus d'une population-mère, pourra être effectuée (Jacquard,1974).

Pour ce faire, les généalogies ont été reconstituées sur une profondeur moyenne de 12 générations en utilisant des registres de population, des registres de mariage et des dictionnaires généalogiques. Après informatisation dans une base de données généalogiques, ces généalogies ont été analysées en utilisant un programme développé à l'UQAC pour identifier les fondateurs. La procédure de reconstitution et d'analyse des généalogies a été décrite en détails dans De Braekeleer et al. (1991).

Trois groupes-témoins ont ensuite été créés à l'aide du fichier de population. La sélection de ces groupes témoins s'est effectuée sur la base du lieu et de la date de mariage des parents des enfants atteints (à \pm trois mois près), et sur le statut socioéconomique des couples qui a été défini à priori par la profession de l'homme. On a utilisé ces groupes-témoins pour l'étude de la consanguinité et de la parenté (De Braekeleer et Larochelle 1990). 
CHAPITRE III

RÉSULTATS 


\section{III.1 Histoires cliniques des enfants atteints}

\section{Cas No. 1}

II s'agit d'une fille née en 1987 d'une mère probablement atteinte de dystrophie myotonique. La grossesse et l'accouchement s'étaient déroulés normalement.

A la naissance elle pesait $3,292 \mathrm{~kg}$, mesurait $49 \mathrm{~cm}$; elle présentait un épicanthus, un strabisme convergent et un ictère. Le fond d'oeil était normal. A sept mois elle fut opérée pour corriger son strabisme. La croissance et le développement sont restés dans les limites de la normale jusqu'à neuf mois environ (poids $7,8 \mathrm{~kg}$; taille $66 \mathrm{~cm})$.

Elle fut hospitalisée en décembre 1988 pour constipation et vomissements. Lors de cette hospitalisation une série de tests montra une acidose métabolique, une hypothyroïdie, une hypocalcémie (calcium: 2,1 mmol/l; $\mathrm{N}: 2,2-2,6 \mathrm{mmol} / \mathrm{l}$ ), une hypokaliémie, une tubulopathie rénale proximale avec décompensation légère, et du rachitisme particulièrement important au niveau des membres inférieurs. Le phosphore sanguin était à $1,0 \mathrm{mmol} / \mathrm{l}$ ( $N$ : 1,2-1,8 $\mathrm{mmol} / \mathrm{l})$ et la phosphatase alcaline à $673 \mathrm{U} / \mathrm{l}(\mathrm{N}: 90-350 \mathrm{U} / \mathrm{l})$. Elle fut traitée au Calcitriol. Le diagnostic de cystinose fut fortement soupçonné. II fut confirmé deux mois plus tard à l'hôpital Ste-Justine de Montréal notamment par un examen ophtalmologique où l'on nota des dépôts de cystine au niveau de la cornée. 
A l'âge de deux ans elle a présenté une subluxation du radius gauche; les examens sanguins effectués à ce moment montrait un calcium à 2,6 $\mathrm{mmol} / \mathrm{l}$, un phosphore à $1,3 \mathrm{mmol} / \mathrm{l}$ et une phosphatase alcaline à $387 \mathrm{U} / \mathrm{l}$. Le traitement à la phosphocystéamine fut commencé en janvier 1990.

En novembre 1990 elle fut de nouveau hospitalisée pour une otite moyenne, une bronchite asthmatique et déshydratation. En juin 1991 l'enfant a fait une pneumonie.

Durant les quatre premières années d'évolution de la maladie la fonction rénale mesurée par les taux sanguins d'urée et de créatinine est restée normale. Une anémie s'est installée progressivement. En mars 1989 les globules rouges étaient à 3,3 x1012/I (N: 4,2-5,4 x 1012/), l'hémoglobine à $88 \mathrm{gr} / \mathrm{l}(\mathrm{N}: 106-152$ $\mathrm{gr} / \mathrm{l})$, l'hématocrite à $0,27(\mathrm{~N}: 0,33-0,43)$. Depuis le début du traitement à la phosphocystéamine une amélioration de son anémie a été constatée (GR: $5,02 \times 10^{12} / \mathrm{Hb}: 139 \mathrm{~g} / \mathrm{l} ; \mathrm{Ht}: 0,40$ ). Les derniers résultats sanguins en date du mois d'août 1991 montraient une urée à $2,5 \mathrm{mmol} / \mathrm{l}$ ( $\mathrm{N}: 2-8 \mathrm{mmol} / \mathrm{l})$, une créatinine à $53 \mu \mathrm{mol} / \mathrm{l}(\mathrm{N}: 40-140$ $\mu \mathrm{mol} / \mathrm{l})$, un calcium à $2,5 \mathrm{mmol} / \mathrm{l}$, un phosphore à $0,8 \mathrm{mmol} / \mathrm{l}$, une phosphatase alcaline à $392 \mathrm{U} / \mathrm{l}$ et un potassium à $2,7 \mathrm{mmol} / \mathrm{l}(\mathrm{N}: 3,5-$ $5,3 \mathrm{mmol} / \mathrm{l})$. 


\section{Cas No. 2}

II s'agit d'un garçon né en 1982. L'enfant était normal à la naissance; il pesait $3,15 \mathrm{~kg}$ et mesurait $53 \mathrm{~cm}$. Vers l'âge de six mois il présenta de la glycosurie, de l'acidose tubulaire et un syndrome de Fanconi (calcium: 2,3 mmol/l; phosphore: 1,2 mmol/l; phosphatase alcaline: $110 \mathrm{U} / \mathrm{l})$. Une cystinose fut soupçonnée mais l'examen ophtalmologique ne montra pas de cristaux de cystine. En janvier 1983, une biopsie cutanée montra une grande concentration de cystine dans les cellules. II présenta alors plusieurs épisodes de déshydratation et d'acidose. Entre 1983 et 1986 l'enfant présenta une anémie chronique (GR: $5,72 \times 10^{12} / \mathrm{l} ; \mathrm{Hb}: 95 \mathrm{~g} / \mathrm{l} ; \mathrm{Ht}: 0,30$ ) et une hypokaliémie ( $\mathrm{K}: 3,3 \mathrm{mmol} / \mathrm{l})$. Sa fonction rénale était normale (urée: $6,8 \mathrm{mmol} / \mathrm{l}$; créatinine: $80 \mu \mathrm{mol} / \mathrm{l})$. Son taux sérique de calcium et de phosphore restait dans les limites de la normale $(2,5 \mathrm{mmol} / \mathrm{l}$ et 1,2 $\mathrm{mmol} / \mathrm{l}$ respectivement).

En avril 1986 l'enfant fut hospitalisé parce qu'il souffrait d'anémie, d'acidose et d'hypokaliémie. Entre le mois de juin 1986 et le mois de septembre 1988 il a été hospitalisé à huit reprises pour des épisodes de vomissements, de déshydratation, de constipation et de déséquilibres électrolytiques. Durant cette période il reçut plusieurs transfusions. Son taux sanguin de calcium s'est maintenu dans les valeurs normales alors qu'on assistait (en septembre 1988) à une augmentation de l'urée sanguine $(12,3 \mathrm{mmol} / \mathrm{l})$ tandis que son taux de créatinine se maintenait dans la normale $(126 \mu \mathrm{mol} / \mathrm{l})$.

Un traitement à la cystéamine fut commencé en juin 1989. Depuis lors on assista à la stabilisation de son état. Les analyses 
biochimiques réalisées en septembre 1991 montraient des GR à 4,4 x $1012 /$, une $\mathrm{Hb}$ à $115 \mathrm{~g} / \mathrm{l}$, une $\mathrm{Ht}$ à 0,34 , une urée à $14 \mathrm{mmol} / \mathrm{l}$, une créatinine à $108 \mu \mathrm{mol} / \mathrm{l}$, un calcium à $2,4 \mathrm{mmol} / \mathrm{l}$, un phosphore à 1,3 $\mathrm{mmol} / \mathrm{l}$ et des phosphatases alcalines à 362 unités/l. 


\section{Cas No. 3}

II s'agit d'une fille née en 1983. L'enfant était normal à la naissance; elle pesait $3,292 \mathrm{~kg}$ et mesurait $50 \mathrm{~cm}$; elle présentait un prominauris. La croissance se déroula normalement jusqu'en mai 1984; on nota à ce moment un arrêt de la croissance. En juillet 1984 un rachitisme très prononcé fut confirmé à la radiographie des genoux et des poignets. Le calcium sanguin était à $2,3 \mathrm{mmol} / \mathrm{l}$, le phosphore à $0,7 \mathrm{mmol} / \mathrm{l}$ et la phosphatase alcaline à $482 \mathrm{U} / \mathrm{l}$. Le médecin soupçonna une cystinose et lui fit passer un examen ophtalmologique qui s'avéra normal. Au mois d'août et septembre de la même année elle fut hospitalisée à plusieurs reprises pour acidose métabolique, déshydratation, déséquilibre électrolytique, glycosurie et protéinurie.

On diagnostiqua alors un syndrome de Fanconi ainsi qu'un rachitisme rénal avec de l'hypophosphorémie, une hyperphosphatasémie alcaline et une hypocalcémie. Un frottis de la moelle osseuse fut effectué en septembre 1984 mais ne montra pas de cristaux de cystine. Un traitement au Calcitriol fut alors prescrit.

Elle fut hospitalisée une nouvelle fois à la fin de septembre 1984 pour hyperthermie due à une amygdalite. En septembre 1985 elle fut de nouveau hospitalisée pour hyperthermie; on lui fit alors passer un autre examen ophtalmologique qui ne montra pas de cristaux de cystine. Ce n'est qu'en mars 1988 qu'on découvrit des cristaux de cystine dans la cornée des deux yeux; le diagnostic de cystinose fut alors confirmé. 
En juin 1990 un nouvel examen ophtalmologique montra des cristaux multiples diffus dans la pleine épaisseur des deux cornées. Cette atteinte cornéenne sévère lui causait un léger handicap visuel.

Le traitement à la phosphocystéamine fut commençé en avril 1991. Plusieurs examens sanguins réalisés en 1991 ont permis de constater l'installation d'une anémie chronique. Les examens sanguins réalisés en août 1991 montraient des GR à $2,1 \times 10^{12} /$, une $\mathrm{Hb}$ à $65 \mathrm{~g} / \mathrm{l}$, un $\mathrm{Ht}$ à 0,19 , une urée à $16 \mathrm{mmol} / \mathrm{l}$, une créatinine à 150 $\mu \mathrm{mol} / \mathrm{l}$, un calcium à $2,3 \mathrm{mmol} / \mathrm{l}$, un phosphore à $1,7 \mathrm{mmol} / \mathrm{l}$ et une phosphatase alcaline à $234 \mathrm{U} / \mathrm{I}$.

\section{Cas No. 4}

II s'agit d'une fille née en 1971. L'enfant était normal à la naissance. En janvier 1973 elle présenta de la polydipsie et de la polyurie; le diagnostic de cystinose fut alors envisagé.

Elle fut hospitalisée en février 1973 pour acidose métabolique, rachitisme rénal, glycosurie et syndrome de Fanconi. Le calcium sérique était à $2,5 \mathrm{mmol} / \mathrm{l}$, le phosphore à $1,7 \mathrm{mmol} / \mathrm{l}$ et la phosphatase alcaline à $1504 \mathrm{U} / \mathrm{l}$. Un examen ophtalmologique ne montra pas de cristaux de cystine. Une ponction de moelle osseuse ne s'avéra pas concluante. L'enfant fut alors perdue de vue.

En décembre 1977 une anémie sévère nécessita une transfusion; on nota la présence de cystine en quantité anormale dans les urines. Une insuffisance rénale sévère s'installa en 1978. L'enfant présenta plusieurs épisodes rebelles d'épistaxis. 
Son anémie nécessita encore plusieurs transfusions pendant les cinq premiers mois de 1978. A ce moment il y eut détérioration de l'état général. Elle présenta une dyspnée sévère, de la tachycardie, une hépatomégalie et des oedèmes à godet aux extrémités. Son état ne cessa d'empirer jusqu'à son décès survenu le 30 mai 1978 dû à une insuffisance cardiaque. Les examens sanguins réalisés quelques heures avant son décès montrèrent des GR à $1,5 \times$ $10^{12} / \mathrm{l}$, une $\mathrm{Hb}$ à $45 \mathrm{~g} / \mathrm{l}$, un $\mathrm{Ht}$ à 0,15 , une urée à $52,3 \mathrm{mmol} / \mathrm{l}$ et une créatinine à $776 \mu \mathrm{mol} / \mathrm{l}$. L'autopsie a été refusée.

Elle avait un frère qui était décédé de cystinose à quelques mois. Aucune information complémentaire n'a pu être obtenue concernant cet enfant.

\section{Cas No. 5}

II s'agit d'un garçon né en 1985 par césarienne. La grossesse s'était déroulée normalement. A la naissance il pesait 3,3 $\mathrm{kg}$, mesurait $49 \mathrm{~cm}$ et présentait un ictère. A neuf mois il a eu un traumatisme crânien; il commença à marcher à l'âge de treize mois.

II fut hospitalisé en février 1988; on nota alors un retard staturo-pondéral, de la déshydratation, une polydipsie, une polyurie persistante depuis plus d'un an ainsi que de la constipation. Une cystographie mictionnelle montra un reflux vésico-urétéral droit. A l'âge de deux ans et onze mois il avait un âge osseux de 19 mois. Un diagnostic de cystinose fut envisagé mais l'examen ophtalmologique s'avéra normal. 
En mai 1988 on diagnostiqua un syndrome de Barter. En novembre 1989 il fut hospitalisé à l'hôpital Ste-Justine mais ce n'est qu'en mars 1990 que le diagnostic de cystinose a été confirmé.

En juillet 1990 il a eu une fracture du tiers moyen de la jambe droite; la radiographie faite en septembre 1991 montra qu'elle était en cours de consolidation.

L'enfant est traité au Calcitriol depuis novembre 1990. II ne présente pas d'insuffisance rénale ni d'anémie. Les derniers résultats sanguins datant de août 1991 montaient une urée à 13,9 mmol/l, une créatinine à $134 \mu \mathrm{mol} / \mathrm{l}$, un calcium à $2,5 \mathrm{mmol} / \mathrm{l}$, un phosphore à $1,9 \mathrm{mmol} / \mathrm{l}$ et une phosphatase alcaline à $391 \mathrm{U} / \mathrm{l}$. II est le deuxième enfant d'une famille de deux enfants.

Cas No. 6

II s'agit d'une fille née en 1980 d'un accouchement normal; à la naissance elle pesait $3,04 \mathrm{~kg}$ et mesurait $48 \mathrm{~cm}$. A neuf mois elle souffrait de polydipsie, de polyurie et présentait un arrêt de croissance. Elle commença à marcher à quinze mois; son âge osseux était celui d'un enfant de neuf mois.

En mars 1982, on diagnostiqua un syndrome de Fanconi et un rachitisme rénal; les analyses de sang montraient des GR à 3,5 x $1012 / \mathrm{l}$, une $\mathrm{Hb}$ à $95 \mathrm{~g} / \mathrm{l}$, un $\mathrm{Ht}$ à 0,28 , un calcium à $2,4 \mathrm{mmol} / \mathrm{l}$, un phosphore à $0,9 \mathrm{mmol} / \mathrm{l}$ et une phosphatase alcaline à $897 \mathrm{U} / \mathrm{l}$. Le diagnostic de cystinose fut confirmé à la biopsie de moelle osseuse qui montrait la présence de dépôts de cystine. L'examen 
ophtalmologique montra également des cristaux de cystine dans la cornée. Un traitement au Calcitriol fut commencé.

Entre 1982 et 1987 elle présentait une anémie chronique et fit plusieurs épisodes de tétanie. Une insuffisance rénale commença à s'installer vers juin 1987 mais l'enfant fut perdue de vue en décembre 1987. A ce moment les GR étaient à $2,7 \times 10^{12} / \mathrm{l}$, l'Hb à 80 g/l, l'Ht à 0,24 , la créatinine à $190 \mu \mathrm{mol} / \mathrm{l}$, le calcium à $2,3 \mathrm{mmol} / \mathrm{l}$, le phosphore à $1,8 \mathrm{mmol} / \mathrm{l}$ et la phosphatase alcaline à $146 \mathrm{U} / \mathrm{l}$.

\section{Cas No. 7}

Ce garçon né en 1981 a été vu pour la première fois à la clinique de pédiatrie de Chicoutimi en juillet 1989. A ce moment, le diagnostic de cystinose avec insuffisance rénale secondaire et syndrome de Fanconi avait déjà été posé. L'enfant souffrait de polyurie massive et sa cornée était blanchâtre. Un traitement au $\mathrm{KCl}$ et au Calcitriol fut instauré. En septembre 1989 il a eu une dialyse péritonéale. Les résultats de laboratoire réalisés avant la dialyse montraient des GR à $3,7 \times 10^{12} / \mathrm{l}$, une $\mathrm{Hb}$ à $96 \mathrm{~g} / \mathrm{l}$, un $\mathrm{Ht}$ à 0,29 , une urée à $14,3 \mathrm{mmol} / \mathrm{l}$, une créatinine à $277 \mu \mathrm{mol} / \mathrm{l}$, un calcium à 2,5 mmol/l, un phosphore à $1,4 \mathrm{mmol} / \mathrm{l}$ et une phosphatase alcaline à 646 $\mathrm{U} / \mathrm{I}$.

En 1990 on nota l'apparition d'une anémie chronique et l'aggravation de son insuffisance rénale. En août 1990 les GR étaient à $2,86 \times 1012 / \mathrm{l}, \mathrm{l} \mathrm{Hb}$ à $84 \mathrm{~g} / \mathrm{l}$, l'Ht à 0,25 , l'urée à $20 \mathrm{mmol} / \mathrm{l}$, la créatinine à $343 \mu \mathrm{mol} / \mathrm{l}$, le calcium à $2,5 \mathrm{mmol} / \mathrm{l}$, le phosphore à 1,7 
$\mathrm{mmol} / \mathrm{l}$ et la phosphatase alcaline à $330 \mathrm{U} / \mathrm{l}$. L'enfant n'a plus été revu depuis décembre 1990.

\section{Cas No. 8}

II s'agit d'une fille née en 1980 d'un accouchement normal suite à une grossesse normale. A la naissance elle pesait $3,519 \mathrm{~kg}$ et mesurait $53 \mathrm{~cm}$. A dix-huit mois elle souffrait de polydipsie et de polyurie. La courbe de glycémie provoquée montrait un état prédiabétique.

Ce n'est qu'en octobre 1986 que le diagnostic de cystinose a été confirmé. L'examen ophtalmologique avait montré la présence de cristaux de cystine dans la cornée. Le médecin observa que l'enfant présentait un visage "poupin", des cheveux très blonds, un retard staturo-pondéral la situant au $3^{\text {ième }}$ percentile et de l'insuffisance rénale. En 1987, elle était anémique ( GR: 3,2 x $10^{12} \mathrm{/} ; \mathrm{Hb}: 86 \mathrm{~g} / \mathrm{l} ; \mathrm{Ht}$ : 0,25 ) et son insuffisance rénale s'aggravait (urée: $20 \mathrm{mmol} / \mathrm{l}$; créatinine: $176 \mu \mathrm{mol} / \mathrm{l})$. Son bilan phospho-calcique montrait un calcium à $2,3 \mathrm{mmol} / \mathrm{l}$, un phosphore à $1,5 \mathrm{mmol} / \mathrm{l}$ et une phosphatase alcaline à $304 \mathrm{U} / \mathrm{l}$.

En janvier 1988 le diagnostic de rachitisme rénal fut posé; elle fut traitée au Calcitriol. La dialyse péritonéale fut commencée en mai 1988. Avant la dialyse l'urée était à $12,1 \mathrm{mmol} / \mathrm{l}$ et la.créatinine à $352 \mu \mathrm{mol} / \mathrm{l}$. Une hypothyroïdie fut diagnostiquée en octobre 1988. Elle a aussi présenté deux péritonites secondaires à la dialyse péritonéale et son anémie a nécessité des.transfusions à 
plusieurs reprises. Malgré la dialyse, on constata une aggravation de l'insuffisance rénale, ce qui amena la décision de transplantation rénale.

Elle a reçu une greffe rénale en novembre 1989. Avant l'intervention chirurgicale les analyses biochimiques montraient des GR à $1,52 \times 10^{12} / \mathrm{l}$, une $\mathrm{Hb}$ à $46 \mathrm{~g} / \mathrm{l}$, un $\mathrm{Ht}$ à 0,13 , une urée à 12,9 mmol/l et une créatinine à $918 \mu \mathrm{mol} / \mathrm{l}$. Elle quitta l'hôpital vers la mi-décembre. Elle a été mise sous traitement anti-rejet. Jusqu'en mai 1990 on assista à une normalisation de sa fonction rénale (urée: 8,1 $\mathrm{mmol} / \mathrm{l}$; créatinine: $131 \mu \mathrm{mol} / \mathrm{l})$. En juin 1990 elle présenta des signes de rejet et une infection à cytomégalovirus pour lesquels elle fut hospitalisée. Entre juin et décembre 1990 on assista à une réinstallation progressive de son anémie (GR: $3,59 \times 10^{12} / \mathrm{l} ; \mathrm{Hb}: 91$ $\mathrm{gr} / \mathrm{l} ; \mathrm{Ht}: 0,28)$ alors que sa fonction rénale restait dans les limites de la normale (urée: 10,5 mmol/l; créatinine: $123 \mu \mathrm{mol} / \mathrm{l}$ ).

Elle fit un deuxième épisode de rejet en janvier 1991 et un troisième en février 1991. Elle fut hospitalisée de nouveau en juin 1991; elle souffrait alors de pneumonie bilatérale, d'hypotension, d'insuffisance surrénalienne et d'anémie (GR: $2,98 \times 10^{12} / \mathrm{H} ; \mathrm{Hb}: 82$ $\mathrm{g} / \mathrm{l} ; \mathrm{Ht}: 0,25$ ). Sa fonction rénale montrait certains signes de décompensation (urée: $15 \mathrm{mmol} / \mathrm{l}$; créatinine: $133 \mu \mathrm{mol} / \mathrm{l}$ ). Depuis que le traitement au Calcitriol a été instauré la calcémie et la phosphorémie se sont maintenues à des valeurs normales sauf pour la phosphorémie pour laquelle les valeurs se sont effondrées après la greffe rénale, passant de $2,5 \mathrm{mmol} / \mathrm{l}$ à $0,8 \mathrm{mmol} / \mathrm{l}$. 
Les principales caractéristiques rencontrées chez les enfants du SLSJ sont résumées dans le tableau 2.1. 
TABLEAU 2.1

Caractéristiques cliniques rencontrées chez 8 des 9 enfants atteints de cystinose au SLSJ

\begin{tabular}{|c|c|c|c|c|c|c|c|c|c|}
\hline $\begin{array}{l}\text { Caractéristiques } \\
\text { cliniques }\end{array}$ & 1 & 2 & 3 & 4 & 5 & 6 & 7 & 8 & Ratio \\
\hline $\begin{array}{l}\text { Né d'un accouchement } \\
\text { normal }\end{array}$ & + & 0 & 0 & 0 & & + & 0 & + & $3 / 8$ \\
\hline Né par césarienne & & & & & + & & & & $1 / 8$ \\
\hline Ictère & + & & & & & & & & $1 / 8$ \\
\hline Epicanthus & + & + & & & & & & & $2 / 8$ \\
\hline Syndrome de Fanconi & & + & + & + & & + & + & & $5 / 8$ \\
\hline Rachitisme rénal & + & & + & + & + & + & & + & $6 / 8$ \\
\hline Tubulopathie & + & & & & & & & & $1 / 8$ \\
\hline Insuffisance rénale & & & & + & & + & + & + & $4 / 8$ \\
\hline Polyurie & & & & + & + & + & + & + & $5 / 8$ \\
\hline Glycosurie & & + & + & + & & & & & $3 / 8$ \\
\hline Protéinurie & & & + & & & & & & $1 / 8$ \\
\hline Polydipsie & & & & + & + & + & & + & $4 / 8$ \\
\hline Acidose & + & + & + & + & & & & & $4 / 8$ \\
\hline $\begin{array}{l}\text { Déséquilibres } \\
\text { électrolytiques }\end{array}$ & + & + & + & & & & & & $3 / 8$ \\
\hline Vomissements & + & + & & & & & & & $2 / 8$ \\
\hline Déshydratation & + & + & + & & + & & & & $4 / 8$ \\
\hline Hypophosphorémie & & & + & & & & & & $1 / 8$ \\
\hline $\begin{array}{l}\text { Hyperphosphatasémie } \\
\text { alcaline }\end{array}$ & & & + & & & & & & $1 / 8$ \\
\hline Hypokaliémie & + & + & & & & & & & $2 / 8$ \\
\hline
\end{tabular}


TABLEAU 2.1 (suite)

\begin{tabular}{|c|c|c|c|c|c|c|c|c|c|}
\hline $\begin{array}{l}\text { Caractéristiques } \\
\text { cliniques }\end{array}$ & 1 & 2 & 3 & 4 & 5 & 6 & 7 & 8 & Ratio \\
\hline Hypocalcémie & + & & + & & & + & & + & $4 / 8$ \\
\hline Hypothyroïdie & + & & & & & & & + & $2 / 8$ \\
\hline Anémie & & & + & + & & + & + & + & $5 / 8$ \\
\hline Constipation & + & + & & & + & & & & $3 / 8$ \\
\hline Retard staturo-pondéral & & & + & + & & + & & + & $4 / 8$ \\
\hline $\begin{array}{l}\text { Cristaux de cystine dans } \\
\text { la cornée }\end{array}$ & + & & + & & & + & + & + & $5 / 8$ \\
\hline Handicap visuel & & & + & & & & & & $1 / 8$ \\
\hline Strabisme convergent & + & & & & & & & & $1 / 8$ \\
\hline Hépatomégalie & & & & + & & & & & $1 / 8$ \\
\hline Tétanie & & & & & & & + & & $1 / 8$ \\
\hline Transfusions & & + & & + & & & & + & $3 / 8$ \\
\hline Traitement au Calcitriol & + & & + & & + & + & + & + & $6 / 8$ \\
\hline $\begin{array}{l}\text { Traitement à la } \\
\text { (Phospho)cystéamine }\end{array}$ & + & + & + & & & & & & $3 / 8$ \\
\hline Dialyse péritonéale & & & & & & & + & + & $2 / 8$ \\
\hline Péritonite & & & & & & & & + & $1 / 8$ \\
\hline Transplantation rénale & & & & & & & & + & $1 / 8$ \\
\hline Décès & & & & + & & & & & $1 / 8$ \\
\hline
\end{tabular}

+) caractéristique présente

o) aucune donnée 


\section{III.2 Épidémiologie et génétique}

III.2.1 Incidence de la cystinose au SLSJ

Entre 1971 et 1990 il y a eu 95514 naissances au SLSJ. L'incidence de la cystinose est de 1 pour 11939 naissances. L'incidence a été calculée à partir de 8 enfants car le dernier était né à Sacré Coeur ( Côte Nord).

Le taux de porteurs est de 1 pour 55 habitants.

III.2.2 Distribution spatiale des lieux de naissance des enfants atteints et de leurs parents

La figure 2.1 montre la distribution des lieux de naissance des enfants atteints. Deux sont nés à Chicoutimi, un à Jonquière, trois à Lac à la Croix, un à Notre-Dame du Rosaire, un à Sacré Coeur et un à St-Honoré.

En ce qui concerne les lieux de naissance des parents, on constate qu'ils sont presque tous originaires de la région. Deux d'entre eux sont nés à Chicoutimi, deux à Dolbeau, deux à Hébertville, trois à Jonquière, deux à Lac à la Croix, deux à Sacré-Coeur, deux à St-Honoré et le dernier est inconnu car la personne a été adoptée.

Les recherches généalogiques ont dénombré 61 fondateurs et 56 ancêtres communs aux porteurs obligatoires. Les distributions de leurs lieux d'origine respectifs sont décrit dans les tableaux 2.2 et 2.3 . 
FIGURE 2.1 :Distribution des lieux de naissance des enfants et des porteurs obligatoires de cystinose du Saguenay-Lac-St-Jean. 
LA REgION DU SAGUENAY LAC-SAINT-JEAN

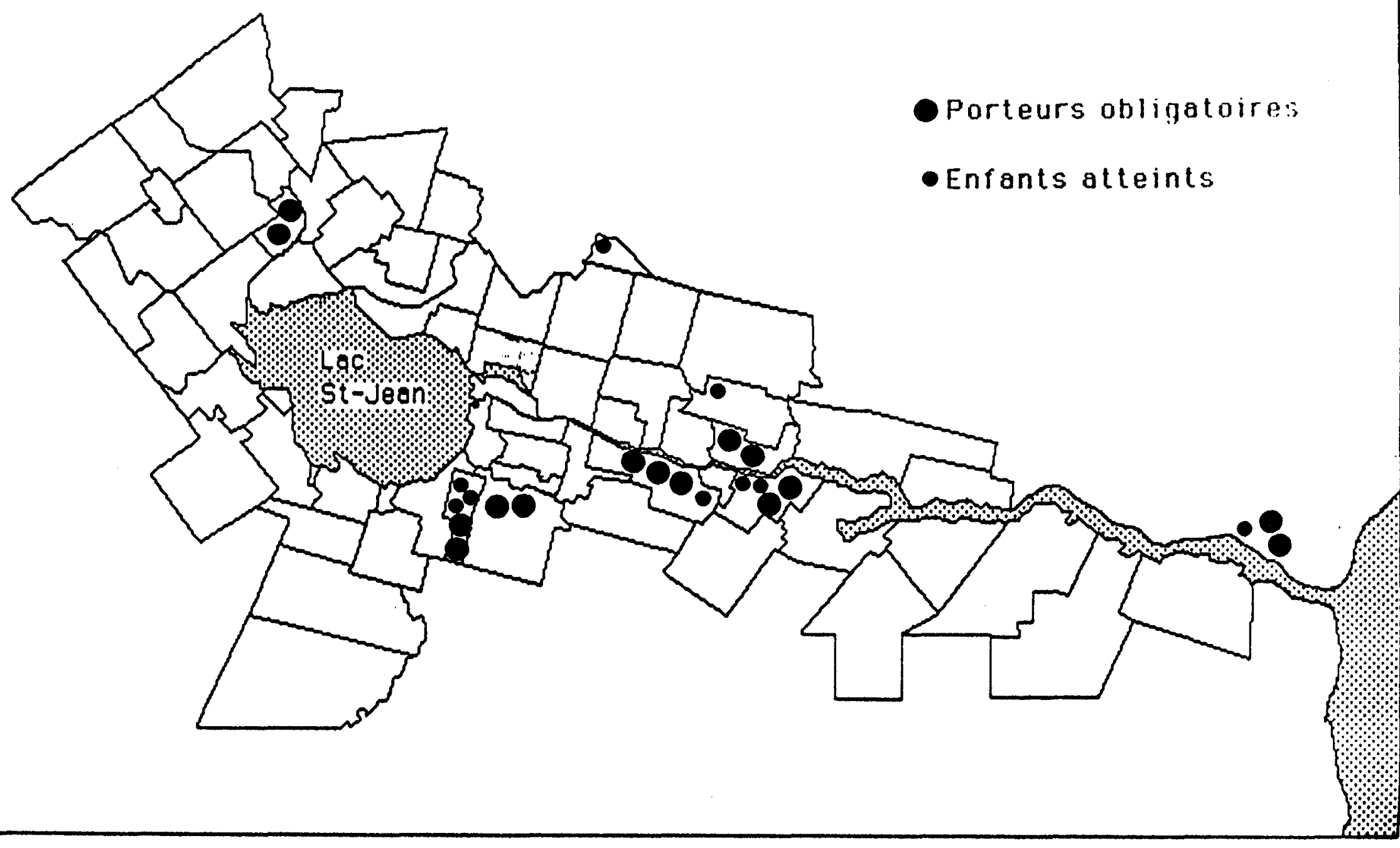


TABLEAU 2.2

Distribution des lieux d'origine des fondateurs communs aux porteurs obligatoires

\begin{tabular}{|l|l|}
\hline Lieux de naissance & Nombre \\
\hline Anjou & 1 \\
\hline Angoumois & 1 \\
\hline Aunis & 10 \\
\hline Beauce & 1 \\
\hline Bretagne & 1 \\
\hline Brie & 1 \\
\hline Limousin & 1 \\
\hline Normandie & 14 \\
\hline Maine & 3 \\
\hline Massachusetts & 1 \\
\hline Paris & 1 \\
\hline Perche & 14 \\
\hline Périgord & 1 \\
\hline Picardie & 1 \\
\hline Poitou & 4 \\
\hline Saintonge & 3 \\
\hline Origine inconnue & 61 \\
\hline Total & \\
\hline
\end{tabular}


TABLEAU 2.3

Distribution des lieux d'origine des ancêtres communs aux porteurs obligatoires

\begin{tabular}{|l|l|}
\hline Lieux de naissance & Nombre \\
\hline Ange Gardien & 4 \\
\hline Baie Saint-Paul & 4 \\
\hline Beauport & 1 \\
\hline Beaupré & 7 \\
\hline Château Richer & 7 \\
\hline Charlesbourg & 1 \\
\hline Ile d'Orléans & 1 \\
\hline Lachine & 1 \\
\hline Lauzon & 1 \\
\hline Montréal & 3 \\
\hline Neuville & 2 \\
\hline Petite Rivière & 2 \\
\hline Québec & 17 \\
\hline Sillery & 2 \\
\hline Tilly & 2 \\
\hline Origine inconnue & 1 \\
\hline Total & 56 \\
\hline
\end{tabular}




\section{III.2.3 Consanguinité et parenté}

Le coefficient moyen de consanguinité calculé pour les sept ascendances de la région du SLSJ donne une valeur égale à $26,51 \times 10^{-}$ 4. comparativement à une valeur moyenne de $31,62 \times 10^{-4}$ pour les trois groupes témoins (voir tableau 2.4). On a trouvé deux mariages consanguins dont un est du type 3:3 chez le groupe atteint de cystinose. On a également trouvé deux mariages consanguins dont un 2:2 et un autre 4:4 chez le groupe-témoin numéro 2 .

Le coefficient moyen de parenté également calculé pour les sept ascendances est égal à $2,21 \times 10^{-4}$ dans le groupe cystinose comparativement à $0,76 \times 10^{-4}$ pour les trois groupes-témoins. 


\section{TABLEAU 2.4}

Coefficients moyens de consanguinité et de parenté chez les enfants atteints de cystinose et des groupestémoins au SLSJ.

\begin{tabular}{|c|c|c|c|}
\hline Groupes & $\begin{array}{l}\text { Coefficient moyen } \\
\text { de consanguinité } \\
\left(\times 10^{-4}\right)\end{array}$ & $\begin{array}{l}\text { Coefficient } \\
\text { de parenté } \\
\left(\times 10^{-4}\right)\end{array}$ & moye \\
\hline Cystinose & 26,51 & 2,21 & \\
\hline Témoin 1 & 0,0 & 1,4 & \\
\hline Témoin 2 & 94,87 & 0,23 & \\
\hline Témoin 3 & 0,0 & 0,64 & \\
\hline
\end{tabular}


CHAPITRE IV

\section{DISCUSSION}


Au point de vue clinique on constate que le phénotype rencontré chez les enfants du SLSJ est très semblable à celui décrit dans la littérature (Tableau 2.5). On constate également que l'atteinte ophtalmique est beaucoup moins sévère dans ce groupe, ce qui avait été constaté dans des études précédentes réalisées au Québec (Richler et al.1991). L'homogénéité de l'ensemble des phénotypes suggère que c'est la même mutation que l'on retrouve chez tous les enfants de la région.

Par contre le ratio garçons/filles est de 4:5 pour le groupe du SLSJ, soit l'inverse de ce que l'on devait s'attendre étant donné que dans cette maladie on retrouve généralement plus de garçons atteints que de filles (Gahl et al.1989).

Si l'on regarde l'incidence de la cystinose au SLSJ qui est de 1 pour 11939 naissances on constate qu'elle est deux fois plus élevée que celle calculée en Bretagne (France) qui est de 1 pour 25 909 naissances, ce qui était considéré comme étant très élevé soit 12.5 fois plus élevée que dans le reste de la France (Bois et al. 1976); de plus l'incidence de la cystinose au SLSJ est au moins 10 fois supérieure à l'incidence mondiale qui est évaluée de 1 pour 100000 à 1 pour 200000 naissances (Gahl et al. 1989). Étant donné le faible échantillonnage de la population étudiée, l'incidence de la cystinose pourrait être aussi basse que $1 / 40.000$ ou aussi élevée que $1 / 7.000$ (intervalle de confiance à 95\%). Étant donné qu'il est impossible de déterminer l'intervalle de confiance des incidences rapportées dans la littérature, aucune comparaison n'est possible. 
Le taux de porteurs pour la région est de 1 pour 55 habitants comparativement à 1 pour 77 habitants en Bretagne (Bois et al. 1976), et de 1 pour 200 habitants dans la population mondiale en général (Gahl et al. 1989).

Le coefficient moyen de consanguinité est moins élevé dans le groupe du SLSJ $\left(26,51 \times 10^{-4}\right)$ que dans celui de la Bretagne $(53 \times$ 10-4). Au SLSJ le coefficient moyen de consanguinité pour les trois groupes-témoins $\left(31,62 \times 10^{-4}\right)$ est légèrement supérieur à celui du groupe atteint; cela suggère que le gène est fréquent dans la population.

En ce qui concerne le coefficient moyen de parenté on constate qu'il est plus élevé dans le groupe atteint $\left(2,21 \times 10^{-4}\right)$ que dans les groupes-témoins $\left(0,76 \times 10^{-4}\right)$; cependant aucun enfant atteint n'est apparenté au degré d'arrière-petits-cousins, ce qui peut être un indice d'un effet fondateur.

Ces données nous portent à croire que la consanguinité rapprochée n'est pas un des facteurs qui ont contribué à l'incidence élevée de cette maladie au SLSJ. Ceci est en opposition avec ce qu'avaient suggéré Richler et al.(1991) et O'Regan (1988), à savoir que le taux élevé de consanguinité chez les canadiens-français est à l'origine du phénotype particulier à cette population.

Quant aux quinze porteurs obligatoires 7 sont natifs du Saguenay et six du Lac-Saint-Jean, ce qui est un indice d'endogamie.

Les recherches généalogiques ont dénombré 61 fondateurs communs aux 15 porteurs obligatoires et 56 ancêtres communs nés à l'extérieur de la région du Saguenay au XVIIième et au XVIIIième siècle. $\mathrm{Ce}$ nombre est relativement restreint et montre que les enfants 
atteints de cystinose sont vraisemblablement issus d'un petit nombre de porteurs originaux.

L'étude de la distribution des lieux de naissance des fondateurs communs montre qu'un grand nombre d'entre eux proviennent du nord-ouest de la France (Tableau 2.2). Les deux régions les plus représentées sont la Normandie (14 fondateurs) et le Perche (14 fondateurs); il est important de préciser ici que la proportion de fondateurs originaires du Perche est grande étant donné que lors des périodes de peuplement de la Nouvelle France cette région a seulement fourni 217 fondateurs contre 1111 pour la Normandie (Charbonneau et al.1987). A elles seules ces deux régions comptent près de $46 \%$ de tous les fondateurs identifiés pour la cystinose. La région de l'Aunis vient au troisième rang avec 10 fondateurs. II est intéressant de noter qu'un fondateur est originaire de la Bretagne; rappelons qu'il y a une concentration élevée d'enfants atteints de cystinose dans cette région. On remarque qu'aucune région de l'est de la France n'est représentée ce qui correspond à la description de Charbonneau et al. en 1987 des lieux d'origine des fondateurs français de cette époque; en effet ce dernier a démontré que lors du peuplement blanc du Québec, les premiers immigrants provenaient en grande partie des provinces du centre et des provinces de l'ouest de la France.

L'étude de la distribution des lieux de naissance des 56 ancêtres communs montre que plus de la moitié (31) de ces individus sont originaires de la ville de Québec et de ses environs incluant la rive sud (Lauzon et Tilly), soit 55\% d'entre eux. Quatorze d'entre eux 
sont originaires de la côte de Beaupré et six de la région de Charlevoix (Tableau 2.3).

Cependant vu qu'un grand nombre d'ancêtres communs sont nés au Québec et que le phénotype observé au Québec est légèrement différent de celui observé ailleurs (atteinte ophtalmique), on ne peut exclure qu'il s'agisse d'une nouvelle mutation qui se serait produite au Québec. Si on accepte l'hypothèse selon laquelle la (les) mutation(s) de cystinose a (ont) été amenée(s) d'Europe au XVIIième siècle, on peut conclure que la diffusion de cette maladie s'est faite selon le modèle de peuplement décrit dans Bouchard et al. (1988), selon lequel les premiers immigrants se seraient tout d'abord installés entre Québec et Montréal. Par la suite, les taux de fécondité élevés ont poussé les gens à émigrer plus loin sur les deux rives du Saint-Laurent, soit vers le Bas-Saint-Laurent et la Gaspésie (rive sud), soit vers la Côte de Beaupré et Charlevoix (rive nord). Plus tard (vers 1838) cette émigration s'est prolongée plus au nord ce qui a donné naissance à la région du Saguenay-Lac-St-Jean. En 1871 la population du SLSJ était composée aux trois quarts d'immigrants originaires de Charlevoix (Bouchard et De Braekeleer, 1991).

Si l'on regarde l'ensemble des données épidémiologiques de la cystinose au SLSJ on observe un modèle à peu près semblable à celui des cinq maladies récessives les plus répandues dans cette région (De Braekeleer,1991). 


\section{TABLEAU 2.5}

Comparaison entre les caractéristiques cliniques trouvées dans la littérature et celles des patients du SLSJ

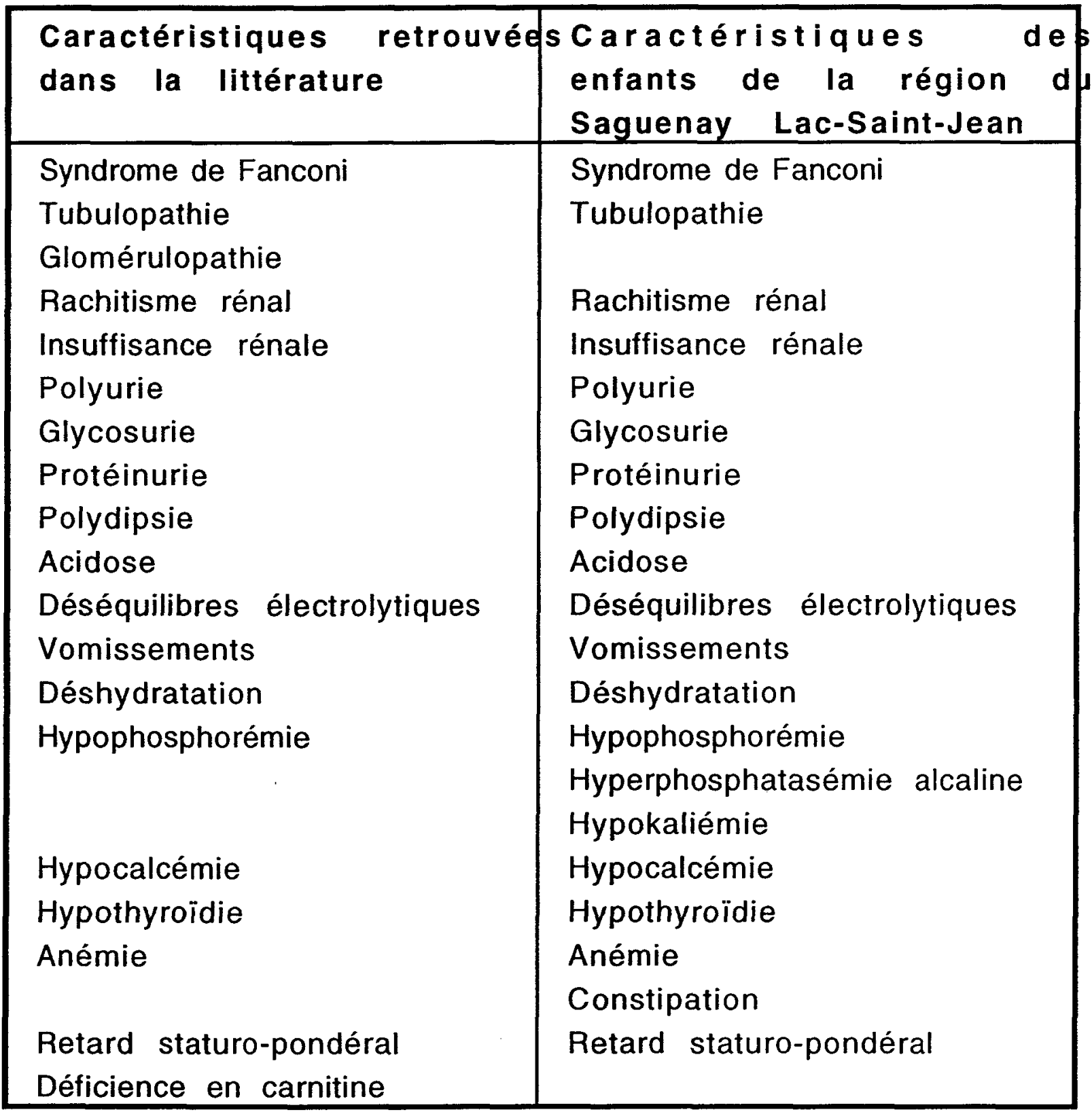


TABLEAU 2.5 (suite)

\begin{tabular}{l|l}
$\begin{array}{l}\text { Hépatosplénomégalie } \\
\text { Photophobie }\end{array}$ & $\begin{array}{l}\text { Hépatomégalie } \\
\text { Tétanie }\end{array}$ \\
$\begin{array}{l}\text { Dépigmentation de la rétine } \\
\text { Cristaux au niveau de la cornée } \\
\text { Handicap visuel }\end{array}$ & $\begin{array}{l}\text { Cristaux au niveau de la cornée } \\
\text { Handicap visuel }\end{array}$
\end{tabular}




\section{CONCLUSION}

Comme il a été démontré précédemment, les enfants atteints de cystinose au SLSJ présentent un tableau clinique très homogène. De plus l'atteinte ophtalmique est moins sévère chez ces enfants que chez ceux qui ne sont pas d'origine canadienne-française; ce qui vient appuyer la thèse que ce soit la même mutation du gène qui est présente dans la région.

L'incidence de la cystinose au SLSJ est étonnamment élevée. Elle surpasse de loin celle de la région de Bretagne en France qui pourtant prenait le premier rang mondial pour l'incidence calculée de cette maladie. Le fait que le coefficient moyen de consanguinité soit nettement moins élevé dans le groupe du SLSJ que dans le groupe de la Bretagne suggère une fréquence du gène beaucoup plus élevée dans la population du SLSJ.

L'absence de parenté proche et le nombre relativement restreint de fondateurs laisse entendre que cette maladie a été introduite par effet fondateur. Suite à l'étude des lieux d'origine de ces fondateurs on a pu constater que l'apport génétique de la région de Bretagne ( 1 fondateur) fut très minime pour cette maladie comparativement aux régions du Perche et de la Normandie.

Bien qu'il apparaisse évident que cette maladie fut amenée d'Europe au Xllième siècle, l'ensemble des données recueillies lors de cette étude suggèrent cependant que le phénotype observé au SLSJ puisse être le résultat d'une mutation qui se serait produite au 
Québec. De plus cette mutation aurait vraisemblablement suivi un modèle de diffusion similaire à plusieurs autres maladies récessives répandues dans cette région. 


\section{BIBLIOGRAPHIE}

Abderhalden E. (1903) Familiare cystindiathese. Z Physio Chem 38: 557-564.

Bois E., Feingold J., Frenay P., Briard M.L. (1976) Infantile cystinosis in France: Genetics, incidence, geographic distribution. J Med Genet 13: $434-438$.

Boman H., Schneider J.A. (1981) Prenatal diagnosis of nephropathic cystinosis. Acta Paediatr Scand 70: 389-393.

Bouchard G., Laberge C., Scriver C.R. (1988) Reproduction démographique et transmission génétique dans le nord-est de la province de Québec $\left(18^{\mathrm{e}}-20^{\mathrm{e}} \mathrm{s}\right.$.). Europ J Population/ Revue européenne de démographie 4: 39-67.

Bouchard G., De Braekeleer M. (1991) Histoire d'un Génôme. Presses de l'Université du Québec.

Cogan D.G., Kuwabara T. (1960) Ocular pathology of cystinosis. Arch Ophthalmol 63: 51-57.

De Braekeleer M., Dionne C., Gagné C., Julien P., Brun D., Murphy M.R.V., Lupien P.J. (1991) Founder effect in familial hyperchylomicronemia among French Canadians of Quebec. Hum Hered 41: 168-173.

De Braekeleer M., Larochelle J. (1990) Genetic epidemiology of hereditary tyrosinemia in Quebec and in Saguenay-Lac-St-Jean. Am J Hum Genet 47: 302-307.

Ehrenreich B.A., Cohn Z.A. (1969) The fate of peptides pinocytosed by macrophages in vitro. $J$ Exp Med 129: 227-245.

Fanconi G. (1936) Der fruhinfantile nephrotish-glycosurische Zwergwuchs mit hypophosphatamischer Rachitis. Jahrb Kinderheilk 146: $299-303$. 
Gahl W.A., Dorfmann A., Evans M.I., Karson E.M., Landsberger F.J., Fabro S.E., Schulman J.D. (1985) Chorionic biopsy in the prenatal diagnosis of nephropathic cystinosis. In: Fraccaro M., Simmoni G., Brampti B. (eds): First Trimester Fetal Diagnosis. Berlin, Springer-Verlag, p.260-278.

Gahl W.A., Reed G.F., Thoene J.G., Schulman J.D., Rizzo W.B. Jonas A.J., Denman D.W., Schlesselman J.J., Corden B.J., Schneider J.A. (1987) Cysteamine therapy for children with nephropathic cystinosis. $N$ Engl J Med 316: 971-977.

GahI W.A., Bernar J., Bernardini I., Dalakas M., Rizzo W.B., Harper G.S., Hoeg J.M., Hurko O. (1988) Oral carnitine replacement in children with cystinosis and renal Fanconi syndrome.J Clin Invest 81: 549-560.

Gahl W.A., Thoene J.G., Schneider J.A., Kaiser-Kupfer M.I., Kuwabara T. (1988) Cystinosis: Progress in a prototypic disease. Ann Intern Med 109: 557-569.

Gahl W.A., Renlund M., Thoene J.G. (1989) Cystinosis and sialic acid storage disorders. In: Scriver C.R., Beaudet A.L., Sly W.S., Valle D. (eds.). The metabolic basis of inherited disease. New York, McGraw Hill, p. 2619-2647.

Gahl W.A., Bashan N., Tietze F., Schulman J.D. (1984) Lysosomal cystine countertransport in heterozygotes for cystinosis. Am $\mathrm{J}$ Hum Genet 36: 277-282.

Gahl W.A., Schneider J.A., Thoene J.G., Chesney R. (1986) Course of nephropathic cystinosis after age 10 years. J Pediatr 109: 605-608.

Goldmann H., Scriver C.R., Aaron K., Delvin E., Canlos Z. (1971) Adolescent cystinosis: Comparisons with infantile and adult forms. Pediatrics 47: 979-988.

Greene A.A., Jonas J.J., Harms E., Smith M.L., Pellett O.L., Bump E.A., Miller A.L., Schneider J.A. (1985) Lysosomal cystine storage in cystinosis and mucolipidosis type II. Pediatr Res 19: 1170-1174.

Gretz N., Manz F., Augustin R. (1982) Survival time in cystinosis. A collaborative study. Proc Europ Dial Transplant Assoc 19: 582-589. 
Holtzapple P.G., Genel M., Yakovac W.C., Hummeler K., Segal S. (1969) Diagnosis of cystinosis by rectal biopsy. $N$ Engl J Med 281: 143-145.

Jacquard A. (1974) Génétique des populations humaines. Presses Universitaires de France, Paris.

Lubec G., Nauer G., Pollack A. (1983) Non-invasive diagnosis of cystinosis by infra-red spectroscopy of hair. Lancet II: 623.

Manz F., Gretz N. (1985) Cystinosis in the Federal Republic of Germany: Coordination and analysis of data. J Inherited Metab Dis 8: 2-4.

Pisoni R.L., Thoene J.G., Christensen H.N. (1985) Detection and characterization of carrier-mediated cationic amino acid transport in lysosomes of normal and cystinotic human fibroblasts. Role in therapeutic cystine removal. J Biol Chem 206: 4791-4798.

Richler M., Milot J., Quigley M., O'Regan S. (1991) Ocular manifestations of nephropathic cystinosis: The french-canadian experience in a genetically homogeneous population. Arch Ophthalmol 109: 356-362.

Schneider J.A., Verroust F.M., Kroll W.A., Garvin A.J., Horger E.O., Wong V.G., Spear G.S., Jacobson C., Pellett O.L., Becker F.L.A. (1974) Prenatal diagnosis of cystinosis. N Engl J Med 290: 878-882.

Schneider J.A., Bradley K.H., Seegmiller J.E. (1967) Increased cystine in leukocytes from individuals homozygous for cystinosis. Science 157: 1321-1322.

Schneider J.A., Bradley K.H., Seegmiller J.E. (1968) Transport and intracellular fate of cysteine- $S$ in leukocytes from normal subjects and patients with cystinosis. Pediatr Res 2: 441-450.

Schneider J.A., Wong V., Bradley K.H., Seegmiller J.E. (1968) Biochemical comparisons of the adult and childhood forms of cystinosis. N Engl J Med 279: 1253-1257.

Schulman J. (1989) Cystinosis: Genetics of Kidney Disorders. Prog Clin Biol Res 305: 137-144. 
Schulman J.D., Bradley K.H. (1970) Metabolism of amino acids, peptides and disulfides in the lysosomes of fibroblasts cultured from normal individuals and those with cystinosis. J Exp Med 132: 10901104.

Smolin L.A., Clark K.F., Schneider J.A. (1987) An improved method for heterozygote detection of cystinosis, using polymorphonuclear leukocytes. Am J Hum Genet 41: 266-275.

States B., Blazer B., Harris D., Segal S. (1975) Prenatal diagnosis of cystinosis. J Pediatr 87: 558-562.

Steinherz R., Raiford D., Mittal K.K., Schulman J.D. (1981) Association of certain human leukocyte antigens with nephropathic cystinosis in the absence of linkage between these loci. Am J Hum Genet 33: 227230 .

Thoene J.G., Oshima R.G., Crawhall J., Olson D., Schneider J.A. (1976) Cystinosis: Intracellular cystine depletion by aminothiols in vitro and in vivo. J Clin Invest 58: 180-189.

Wong V.G. (1973) The eye and cystinosis. In: Schulman J.D. (ed.). Cystinosis. Washington, D.C., DHEW Publication no (NIH) 72-249, Government printing Office, p. 23-35. 


\section{CONCLUSION GÉNÉRALE}

Bien que peu connus le syndrome de Zellweger et la cystinose sont bien présents dans la population du SLSJ et ce à des incidences très élevées. Les résultats obtenus au cours de cette étude viennent rajouter ces deux maladies à la liste déjà longue des maladies autosomales récessives à incidence élevée dans la région.

Le nombre d'enfants atteints de cystinose et du syndrome de Zellweger est particulièrement élevé et ces concentrations sont présentement les plus élevées au niveau mondial ce qui était ignoré jusqu'à ce jour. Les taux de porteurs calculés font également réfléchir quant à la fréquence de certains gènes dans la région.

Comme il a été démontré auparavant par Bouchard et De Braekeleer (1991), les génopathies retrouvées dans cette région ne sont pas imputables à une forte consanguinité mais elles sont le résultat d'un mélange d'effet fondateur et d'endogamie. C'est tout à fait ce qu'on a pu observer au cours de cette étude; en effet le coefficient moyen de consanguinité pour le syndrome de Zellweger est nul et il n'est pas très élevé pour la cystinose; et la plupart des porteurs obligatoires sont originaires de la région. Quant à l'effet fondateur la parenté éloignée et le petit nombre de fondateurs venus du centre et de l'ouest de la France, ont permis d'en démontrer la présence pour la cystinose.

Cette recherche a aussi permis de mieux connaître ces deux maladies au niveau génétique et épidémiologique tout en mettant en évidence l'origine de leurs présences au SLSJ. 